TRANSACTIONS OF THE

AMERICAN MATHEMATICAL SOCIETY

Volume 348, Number 7, July 1996

\title{
ON A PARABOLIC EQUATION WITH A SINGULAR LOWER ORDER TERM
}

\author{
QI ZHANG
}

\begin{abstract}
We obtain the existence of the weak Green's functions of parabolic equations with lower order coefficients in the so called parabolic Kato class which is being proposed as a natural generalization of the Kato class in the study of elliptic equations. As a consequence we are able to prove the existence of solutions of some initial boundary value problems. Moreover, based on a lower and an upper bound of the Green's function, we prove a Harnack inequality for the non-negative weak solutions.
\end{abstract}

\section{INTRODUCTION}

In recent years, there have been many results in the study of elliptic equations with singular lower order terms in the Kato class. We recall that for $n \geq 3$, a function $V \in L_{l o c}^{1}\left(\mathbf{R}^{n}\right)$ is said to belong to the Kato class $K_{n}$ provided that

$$
\lim _{r \rightarrow 0} \sup _{x} \int_{|x-y|<r} \frac{|V(y)|}{|x-y|^{n-2}} d y=0 .
$$

In their celebrated work [AS], using probabilistic ideas, Aizenman and Simon proved that the class $K_{n}$ is the natural replacement of the Lebesgue class $L^{p}$, with $p>n / 2$, in order for solutions of $-\Delta u+V u=0$ to satisfy a Harnack inequality. As a consequence, they obtained a modulus of continuity for such solutions. Subsequently, using PDE methods, the authors of the paper [CFG] generalized the results in [AS] to elliptic equations in divergence form with bounded, measurable coefficients. In both works an important role was played by an embedding result for the class $K_{n}$ due to Schechter.

In contrast to the elliptic case there is not much investigation (known to the author) on parabolic equations with lower order coefficients in a class parallel to the Kato class. In this paper we will take up this problem. As the reader will see, this task presents some notable differences from the elliptic situation. To clarify the point we mention that it is not clear whether a version of Schechter's embedding theorem holds in the parabolic setting. Therefore, we had to work around this obstacle. We benefited from the ideas in [FS2], in which Fabes and Stroock deduced the Harnack inequality for parabolic equations from lower and upper bounds of the fundamental solutions. We succeeded in obtaining similar lower and upper bounds for the fundamental solutions of the parabolic equations with a singular lower order term in the parabolic Kato class, which is being proposed as a natural generalization

Received by the editors September 26, 1994 and, in revised form, May 28, 1995.

1991 Mathematics Subject Classification. Primary 35K10.

(C)1996 American Mathematical Society 
of the Kato class in the elliptic case. Based on these bounds, a Harnack inequality was established. It is interesting to note that the results of the paper recapture those in $[\mathrm{AS}],[\mathrm{CFG}]$ when one deals with time-independent solutions.

We shall study the parabolic equation

$$
H u(x, t) \equiv \sum_{i, j=1}^{n} \frac{\partial}{\partial x_{i}}\left(a_{i j}(x, t) \frac{\partial u}{\partial x_{j}}\right)(x, t)-V(x, t) u(x, t)-\frac{\partial u}{\partial t}(x, t)=0
$$

in a bounded domain $Q=D \times[0, T] \subset \mathbf{R}^{n+1}$. Here $a_{i j}$ are bounded measurable functions and the matrix $a=\left(a_{i j}(x, t)\right)$ is positive definite uniformly in $(x, t)$. This means the existence of a number $\lambda>1$ such that $\lambda^{-1} I \leq a \leq \lambda I$.

For the function $V$ we will impose the following condition which will be called condition K. Let

$$
\begin{aligned}
& N_{h}(V) \equiv \sup _{x, t} \int_{t-h}^{t} \int_{D}|V(y, s)| \frac{1}{(t-s)^{n / 2}} \exp \left(-\alpha \frac{|x-y|^{2}}{t-s}\right) d y d s, \\
& N_{h}^{*}(V) \equiv \sup _{y, s} \int_{s}^{s+h} \int_{D}|V(x, t)| \frac{1}{(t-s)^{n / 2}} \exp \left(-\alpha \frac{|x-y|^{2}}{t-s}\right) d x d t
\end{aligned}
$$

where $\alpha$ is a fixed positive constant and $V(y, s)$ is regarded as zero when $(y, s)$ is outside of $Q$.

Definition. We say that $V$ satisfies condition $\mathrm{K}$ if

$$
\lim _{h \rightarrow 0} N_{h}(V)=\lim _{h \rightarrow 0} N_{h}^{*}(V)=0
$$

The space of all $L_{l o c}^{1}$ functions satisfying condition $\mathrm{K}$ will be called the parabolic Kato class.

In section 2 we will show that the parabolic Kato class is a natural generalization of the Kato class in the study of elliptic equations. Note that the parabolic Kato class depends on the parameter $\alpha$. For our purpose $\alpha$ can be any positive number so that the following inequality holds:

$$
\Gamma_{0}(x, t ; y, s) \leq \frac{C}{(t-s)^{n / 2}} \exp \left(-\alpha \frac{|x-y|^{2}}{t-s}\right),
$$

for some $C>0$ and $0<t-s \leq T$. Here $\Gamma_{0}$ is the fundamental solution of the unperturbed operator $H_{0}$ defined by

$$
H_{0} u \equiv \sum_{i, j=1}^{n} \frac{\partial}{\partial x_{i}}\left(a_{i j}(x, t) \frac{\partial u}{\partial x_{j}}\right)(x, t)-\frac{\partial u}{\partial t}(x, t) .
$$

Before stating the results of the paper we need to give a few more notations and definitions.

Green's functions. From now on we will use $G(x, t ; y, s)$ and $G_{0}(x, t ; y, s)$ to denote the weak Green's functions of operators $H$ and $H_{0}$ for the initial-Dirichlet 
problem on $Q$ respectively. The precise definition of the weak Green's function is standard and can be found in [A]. The symbols $\nabla_{x} G$ and $\nabla_{y} G$ mean the gradient with respect to the first and the second space variables of $G$ respectively.

Weak solutions. Given $f \in L^{1}(Q)$, a weak solution of $H u=f$ in $Q$ is a function $u$ satisfying:

(a) $u \in C^{0}\left([0, T] ; L^{2}(D)\right) \cap L^{2}\left(0, T ; W^{1,2}(D)\right) ;$

(b) $V u \in L^{1}(Q)$;

(c) $\int_{0}^{T} \int_{D}\left[-A \nabla u \nabla \phi-V u \phi+u \phi_{t}\right] d x d t=\int_{0}^{T} \int_{D} f \phi d x d t$

for all $\phi \in C_{0}^{\infty}(Q)$.

Our main results are

Theorem A. The weak Green's function of $H$ for the initial-Dirichlet problem exists and satisfies the following properties.

(a)

$$
\begin{aligned}
& \sup _{y, s}\|G(., . ; y, s)\|_{L^{p}(Q)} \leq C ; \\
& \sup _{x, t}\|G(x, t ; ., .)\|_{L^{p}(Q)} \leq C, \quad 0<p<(n+2) / n .
\end{aligned}
$$

$$
\begin{aligned}
& \sup _{x, t}\left\|\nabla_{y} G(x, t ; ., .)\right\|_{L^{p}(Q)} \leq C ; \\
& \sup _{y, s}\left\|\nabla_{x} G(., . ; y, s)\right\|_{L^{p}(Q)} \leq C, \quad 0<p<(n+2) /(n+1) .
\end{aligned}
$$

Here the constant $C$ depends on $\lambda, p, Q$ and on $V$ in terms of the rate of convergence of (1.4).

A direct consequence of Theorem $\mathrm{A}$ is the next

Theorem B. Suppose $D$ is a bounded $C^{1}$ domain. Given $f \in C_{0}(\bar{D})$ and $g \in$ $L^{\infty}(Q)$, the following initial boundary value problem has a unique bounded weak solution.

$$
\left\{\begin{array}{l}
H u=g \quad \text { in } \quad D \times[0, T], \\
u=0 \quad \text { in } \quad \partial D \times[0, T], \quad \text { and } \quad \lim _{t \rightarrow 0^{+}} u(x, t)=f(x) .
\end{array}\right.
$$

Theorem C (Harnack inequality). Let $0<\alpha_{2}<\beta_{2}<\alpha_{1}<\beta_{1}<1$ and $\delta \in(0,1)$ be given. Then there are $M>0$ and $R_{0}>0$ such that for all $(x, s) \in \mathbf{R}^{n} \times \mathbf{R}$, all positive $R<R_{0}$ and all non-negative weak solutions $u$ of $H u=0$ in $B(x, R) \times[s-$ $\left.R^{2}, s\right]$, one has

$$
\sup _{Q^{-}} u \leq M \inf _{Q^{+}} u
$$

where $Q^{+}=B(x, \delta R) \times\left[s-\beta_{1} R^{2}, s-\alpha_{1} R^{2}\right]$ and $Q^{+}=B(x, \delta R) \times\left[s-\beta_{2} R^{2}, s-\alpha_{2} R^{2}\right]$.

As we will see in the next section, functions satisfying condition $\mathrm{K}$ are in general more singular than $L^{p, q}$ functions when $\frac{n}{2 p}+\frac{1}{q}<1$. Therefore the theory in [A] of uniformly parabolic equations does not cover the results in this paper. Moreover 
the parabolic Kato class we are proposing reduces to the Kato class in the elliptic case when the function $V$ is independent of $t$.

The main body of the paper is organized as follows. In section 2 we will give a brief discussion of the parabolic Kato class. In section 3 we will give an $L^{p}\left(0, T ; W^{1, p}(D)\right)$ estimate of the Green's function of (1.1) when the lower order coefficient $V$ is an $L^{\infty}$ function. The key argument is to show that the estimate depends on the rate of convergence of $N_{h}(V)+N_{h}^{*}(V)$ to zero when $h$ approaches zero, and not on the $L^{\infty}$ norm of $V$. The proof of Theorem A is presented in section 4. The strategy is to approximate $V$ by a sequence of $L^{\infty}$ functions $V_{k}$. The Green's function of (1.1) is then defined as the limit of the Green's functions of (1.1) when $V$ is replaced by $V_{k}$. Using the estimates in section 3, we will be able to show that the function thus defined is indeed the Green's function. In section 5 a lower and an upper bound of the Green's function is established. Based on these bounds, we will prove Theorem C, i.e. the Harnack inequality, in section 6 .

Note. In a recent work [S], K. Sturm investigated properties of weak solutions of equations similar to (1.1), but with smooth coefficients $\left(a_{i j}\right)$. The weak solution in $[\mathrm{S}]$ is defined by a probabilistic means and is in general different from the weak solution in the distributional sense used in this paper.

\section{ACKNOWLEDGMENT}

The author is greatly indebted to Professor N. Garofalo, who not only suggested the problem but also gave much invaluable advice. Thanks should also go to the referee, who made a number of important suggestions.

\section{The Parabolic Kato Class}

In this section we will provide some further analysis of Condition $\mathrm{K}$ and show that the condition is indeed the natural extension of the elliptic Kato class. Since much of the argument is elementary, we tend to be brief.

Proposition 2.1. The parabolic Kato class strictly contains the space $L^{p, q}(Q)$ with $\frac{n}{2 p}+\frac{1}{q}<1$.

Proof. By Hölder's inequality we have

$$
N_{h}(V) \leq C_{h}\|V\|_{L^{p, q}} \text { and } N_{h}^{*}(V) \leq C_{h}\|V\|_{L^{p, q}} .
$$

Here $C_{h}$ is a constant which depends on $p, q$ and $h$ and which goes to zero when $h \rightarrow 0$. We refer the reader to $[\mathrm{S}]$ for details. q.e.d.

Proposition 2.2. If the function $V$ is independent of $t$, then $V$ belongs to the parabolic Kato class if and only if it belongs to the Kato class in the elliptic case, i.e.

$$
\lim _{r \rightarrow 0} \sup _{x} \int_{B_{r}(x)} \frac{|V(y)|}{|x-y|^{n-2}} d y=0, \quad n \geq 3 .
$$

Proof. By direct calculation, when $n \geq 3$,

$$
\begin{aligned}
& \int_{s}^{s+h} \Gamma_{\alpha}(x, t ; y, s) d t \leq \frac{C}{|x-y|^{n-2}}, \\
& \int_{s-h}^{s} \Gamma_{\alpha}(x, t ; y, s) d s \leq \frac{C}{|x-y|^{n-2}}
\end{aligned}
$$


where

$$
\Gamma_{\alpha}(x, t ; y, s)=\frac{1}{(t-s)^{n / 2}} \exp \left(-\alpha \frac{|x-y|^{2}}{t-s}\right) .
$$

The result of the proposition immediately follows. q.e.d.

Proposition 2.3. Let $V^{\prime} \equiv V(x,-t)$. Then

$$
N_{h}\left(V^{\prime}\right)=N_{h}^{*}(V) .
$$

In this sense $N_{h}^{*}$ is a reflection of $N_{h}$.

Proof. From (1.3)

$$
N_{h}\left(V^{\prime}\right)=\sup _{x, t} \int_{t-h}^{t} \int_{D}|V(y,-s)| \frac{1}{(t-s)^{n / 2}} \exp \left(-\alpha \frac{|x-y|^{2}}{t-s}\right) d y d s .
$$

Making the change of variables $s=-s$ we obtain

$$
\begin{aligned}
N_{h}\left(V^{\prime}\right) & =\sup _{x, t}\left[-\int_{-t+h}^{-t} \int_{D}|V(y, s)| \frac{1}{(t+s)^{n / 2}} \exp \left(-\alpha \frac{|x-y|^{2}}{t+s}\right) d y d s\right] \\
& =\sup _{x, t} \int_{-t}^{-t+h} \int_{D}|V(y, s)| \frac{1}{(s-(-t))^{n / 2}} \exp \left(-\alpha \frac{|x-y|^{2}}{s-(-t)}\right) d y d s \\
& =N_{h}^{*}(V) . \quad \text { q.e.d. }
\end{aligned}
$$

Remark. We note that the parabolic Kato class depends on the constant $\alpha$, which appears in (1.2) and (1.3). This is a new situation that does not happen in the elliptic case.

\section{BAsiC estimates}

Unless stating otherwise we assume that $V$ is an $L^{\infty}$ function throughout this section. With this additional assumption on $V$ the existence and uniqueness of the Green's function $G$ of (1.1) are provided by the standard theory of parabolic equations (see $[\mathrm{A}]$ ). The next three propositions will give the $L^{p}$ estimates of $G$ and its gradient. These estimates will be independent of the $L^{\infty}$ norm of $V$ and will play a critical role in the proof of Theorem A in the next section. We start with Proposition 3.1, which is essentially a special case of Theorem A when the cylinder $Q$ is thin enough.

In this and the following sections we will use $C$ to denote a generic constant that may vary in value. In general $C$ always depends on $p, n$ and $Q$.

Proposition 3.1. Let $G$ be the Green's function of (1.1). There exist $h_{0}>0$ and $C>0$ depending only on $H_{0}, Q$ and the rate of convergence of $N_{h}(V)$ to zero when $h \rightarrow 0$, such that the following statements are true whenever $0<h \leq h_{0}$ :

(a)

$$
\sup _{x, t}\|G(x, t ; ., .)\|_{L^{p}\left(Q_{h, t}^{-}\right)} \leq C, \quad p<\frac{n+2}{n} .
$$

$$
\sup _{x, t}\|\nabla G(x, t ; ., .)\|_{L^{p}\left(Q_{h, t}^{-}\right)} \leq C, \quad p<\frac{n+2}{n+1} .
$$


Here $\nabla$ is applied to the second space variable $y$.

(c)

$$
\int_{D}|G(x, t ; y, s)| d y \leq C .
$$

Here $(y, s) \in Q_{h, t}^{-} \equiv D \times[t-h, t]$.

Proof.

(a) Let

$$
H_{0} u(x, t) \equiv \sum_{i, j=1}^{n} \frac{\partial}{\partial x_{i}}\left(a_{i j} \frac{\partial u}{\partial x_{j}}\right)(x, t)-\frac{\partial u}{\partial t}(x, t)=0 .
$$

Throughout the section we shall use $G(x, t ; y, s)$ and $G_{0}(x, t ; y, s)$ to respectively denote the Green's functions of operators $H$ (defined in (1.1)) and $H_{0}$ on domain $Q=D \times[0, T] \subset \mathbf{R}^{n+1}$. Using the standard parametric method, we have the integral equation:

$$
G(x, t ; y, s)=G_{0}(x, t ; y, s)-\int_{s}^{t} \int_{D} G(x, t ; z, \tau) V(z, \tau) G_{0}(z, \tau ; y, s) d z d \tau .
$$

Hence we know the following is true at least formally:

$$
\begin{aligned}
& G(x, t ; y, s) \\
& \quad=G_{0}(x, t ; y, s)+G_{0} * H G_{0}(x, t ; y, s)+\ldots+G_{0} *\left(H G_{0}\right)^{* k}(x, t ; y, s)+\ldots \\
& \quad \equiv J_{0}(x, t ; y, s)+J_{1}(x, t ; y, s)+\ldots+J_{k}(x, t ; y, s)+\ldots,
\end{aligned}
$$

where $H G_{0}(x, t ; y, s) \equiv H_{x, t} G_{0}(x, t ; y, s)=-V(x, t) G_{0}(x, t ; y, s)$ when $t>s$ and

$$
\begin{aligned}
& G_{0} * H G_{0}(x, t ; y, s) \equiv \int_{s}^{t} \int_{D} G_{0}(x, t ; z, \tau) H G_{0}(z, \tau ; y, s) d z d \tau \\
& \left(H G_{0}\right)^{* k} \equiv H G_{0} * H G_{0} * \ldots * H G_{0} \quad(k \text { times }) .
\end{aligned}
$$

Next, we shall prove that the right-hand side of (3.1) is convergent in the sense of $L^{p}$ norm for an adequate $p$. We will divide the proof into several steps.

Step 1. For $h>0$, let $Q_{h, t}^{-} \equiv D \times[t-h, t]$. For a fixed $(x, t)$ we first give an upper bound of

$$
\begin{aligned}
& \left\|J_{1}(x, t ; ., .)\right\|_{L^{p}\left(Q_{h, t}^{-}\right)} \\
& \quad=\sup \left\{\left|\int_{t-h}^{t} \int_{D} J_{1}(x, t ; y, s) \phi(y, s) d y d s\right| \mid \phi \in C_{0}^{\infty}\left(Q_{h, t}^{-}\right),\|\phi\|_{L^{p^{\prime}}\left(Q_{h, t}^{-}\right)} \leq 1\right\} .
\end{aligned}
$$

First we know from $[\mathrm{A}]$ that

$$
G_{0}(x, t ; y, s) \leq C \frac{1}{(t-s)^{n / 2}} \exp \left(-\alpha \frac{|x-y|^{2}}{t-s}\right)
$$

for a positive constant $\alpha$, which implies

$$
\int_{t-h}^{t} \int_{D} G_{0}(x, t ; z, \tau)|V(z, \tau)| d z d \tau \leq C N_{h}(V) .
$$


Now by (3.2) and by exchanging the order of integrals we have

$$
\begin{aligned}
& \left|\int_{t-h}^{t} \int_{D} J_{1}(x, t ; y, s) \phi(y, s) d y d s\right| \\
& \leq \int_{t-h}^{t} \int_{D} \int_{s}^{t} \int_{D} G_{0}(x, t ; z, \tau)|V(z, \tau)| G_{0}(z, \tau ; y, s)|\phi(y, s)| d z d \tau d y d s \\
& =\int_{t-h}^{t} \int_{D} G_{0}(x, t ; z, \tau)|V(z, \tau)| \int_{t-h}^{\tau} \int_{D} G_{0}(z, \tau ; y, s)|\phi(y, s)| d y d s d z d \tau \\
& \leq \int_{t-h}^{t} \int_{D} G_{0}(x, t ; z, \tau)|V(z, \tau)| d z d \tau \sup _{z, \tau} \int_{t-h}^{\tau} \int_{D} G_{0}(z, \tau ; y, s)|\phi(y, s)| d y d s \\
& \leq N_{h}(V) \sup _{z, \tau} \int_{t-h}^{\tau} \int_{D} G_{0}(z, \tau ; y, s)|\phi(y, s)| d y d s \\
& \leq C N_{h}(V) \sup _{z, \tau}\left\|G_{0}(z, \tau ; ., .)\right\|_{L^{p}\left(Q_{h, \tau}^{-}\right)}\|\phi\|_{L^{p^{\prime}}\left(Q_{h, t}^{-}\right)} .
\end{aligned}
$$

It follows that

$$
\left\|J_{1}(x, t ; . . .)\right\|_{L^{p}\left(Q_{h, t}^{-}\right)} \leq C N_{h}(V) \sup _{z, \tau}\left\|G_{0}(z, \tau ; . .,)\right\|_{L^{p}\left(Q_{h, \tau}^{-}\right)}, \tau \leq t .
$$

Step 2. Since

$$
\begin{aligned}
& \left|\int_{t-h}^{t} \int_{D} J_{1}(x, t ; y, s) V(y, s) d y d s\right| \\
& =\left|\int_{t-h}^{t} \int_{D} \int_{s}^{t} \int_{D} G_{0}(x, t ; z, \tau) V(z, \tau) G_{0}(z, \tau ; y, s) V(y, s) d z d \tau d y d s\right| \\
& \leq\left|\int_{t-h}^{t} \int_{D} G_{0}(x, t ; z, \tau) V(z, \tau) \int_{t-h}^{\tau} \int_{D} G_{0}(z, \tau ; y, s) V(y, s) d y d s d z d \tau\right| \\
& \leq \sup _{z, \tau} \int_{t-h}^{\tau} \int_{D} G_{0}(z, \tau ; y, s)|V(y, s)| d y d s \int_{t-h}^{t} \int_{D} G_{0}(x, t ; z, \tau)|V(z, \tau)| d z d \tau \\
& \leq C^{2} N_{h}^{2}(V),
\end{aligned}
$$

we have, by an easy induction,

$$
\begin{aligned}
\mid \int_{t-h}^{t} & \int_{D} J_{k}(x, t ; y, s) V(y, s) d y d s \mid \\
\quad \leq & \int_{t-h}^{t} \int_{D} \int_{s}^{t} \int_{D}\left|J_{k-1}(x, t ; z, \tau) V(z, \tau) G_{0}(z, \tau ; y, s)\right| d z d \tau|V(y, s)| d y d s \\
\quad & \int_{t-h}^{t} \int_{D}\left|J_{k-1}(x, t ; z, \tau) V(z, \tau)\right| \int_{t-h}^{\tau} \int_{D}\left|G_{0}(z, \tau ; y, s) V(y, s)\right| d y d s d z d \tau \\
& \leq C^{k+1} N_{h}^{k+1}(V)
\end{aligned}
$$


This yields

$$
\begin{aligned}
& \left|\int_{t-h}^{t} \int_{D} J_{k+1}(x, t ; y, s) \phi(y, s) d y d s\right| \\
& \leq\left|\int_{t-h}^{t} \int_{D} \int_{s}^{t} \int_{D} J_{k}(x, t ; z, \tau) V(z, \tau) G_{0}(z, \tau ; y, s) \phi(y, s) d z d \tau d y d s\right| \\
& \leq\left|\int_{t-h}^{t} \int_{D} J_{k}(x, t ; z, \tau) V(z, \tau) \int_{t-h}^{\tau} \int_{D} G_{0}(z, \tau ; y, s) \phi(y, s) d y d s d z d \tau\right| \\
& \leq \sup _{z, \tau} \int_{t-h}^{\tau} \int_{D} G_{0}(z, \tau ; y, s)|\phi(y, s)| d y d s\left|\int_{t-h}^{t} \int_{D} J_{k}(x, t ; z, \tau) V(z, \tau) d z d \tau\right| \\
& \leq \sup _{z, \tau} \int_{t-h}^{\tau} \int_{D} G_{0}(z, \tau ; y, s)|\phi(y, s)| d y d s C^{k+1} N_{h}^{k+1}(V) \\
& \leq \sup _{z, \tau}\left\|G_{0}(z, \tau ; ., .)\right\|_{L^{p}\left(Q_{h, \tau}^{-}\right)}|| \phi \|_{L^{p^{\prime}}\left(Q_{h, t}^{-}\right)} C^{k+1} N_{h}^{k+1}(V) .
\end{aligned}
$$

It follows that

$$
\left\|J_{k+1}(x, t ; ., .)\right\|_{L^{p}\left(Q_{h, t}^{-}\right)} \leq C^{k+1} N_{h}^{k+1}(V) \sup _{z, \tau}\left\|G_{0}(z, \tau ; ., .)\right\|_{L^{p}\left(Q_{h, \tau}^{-}\right)}, \quad \tau \leq t .
$$

Going back to (3.1), we obtain

$$
\|G(x, t ; ., .)\|_{L^{p}\left(Q_{h, t}^{-}\right)} \leq \sum_{k=0}^{\infty} C^{k} N_{h}^{k}(V) \sup _{z, \tau}\left\|G_{0}(z, \tau ; ., .)\right\|_{L^{p}\left(Q_{h, \tau}^{-}\right)}, \quad \tau \leq t .
$$

The right-hand side of (3.6) will be convergent if one has

$$
C N_{h}(V)<1 \text {. }
$$

Since $V$ is in the parabolic Kato class, (3.7) can be satisfied if $0<h \leq h_{0}$ which is chosen sufficiently small. Therefore we have

$$
\sup _{x, t}\|G(x, t ; ., .)\|_{L^{p}\left(Q_{h, t}^{-}\right)} \leq C \sup _{z, \tau}\left\|G_{0}(z, \tau ; ., .)\right\|_{L^{p}(D \times[\tau-h, \tau])} \leq C, \quad \tau \leq t .
$$

This implies (a).

Proof of (b). Formally differentiating both sides of (3.1) with respect to $y$ we obtain

$$
\nabla_{y} G(x, t ; y, s)=\sum_{k=0}^{\infty} \nabla_{y} J_{k}(x, t ; y, s) .
$$

Following the proof of part (a) step by step, we know that the right-hand side of (3.9) is convergent in $L^{p}$ norm when $p<(n+2) /(n+1)$ and that

$$
\sup _{x, t}\|\nabla G(x, t ; . . .)\|_{L^{p}\left(Q_{h, t}^{-}\right)} \leq C \sup _{z, \tau}\left\|\nabla G_{0}(z, \tau ; . . .)\right\|_{L^{p}(D \times[\tau-h, \tau])} \leq C, \quad \tau \leq t .
$$

This proves (b). 
Proof of $(c)$. Our starting point is again (3.1). It is clear that

$$
\int_{D} G_{0}(x, t ; y, s) d y \leq C .
$$

Furthermore, when $0<t-s<h$,

$$
\begin{aligned}
\int_{D}\left|J_{1}(x, t ; y, s)\right| d y & \leq \int_{D} \int_{s}^{t} \int_{D} G_{0}(x, t ; z, \tau)|V(z, \tau)| G_{0}(z, \tau ; y, s) d z d \tau d y \\
& =\int_{s}^{t} \int_{D} G_{0}(x, t ; z, \tau)|V(z, \tau)| \int_{D} G_{0}(z, \tau ; y, s) d y d z d \tau \\
& \leq C \int_{s}^{t} \int_{D} G_{0}(x, t ; z, \tau)|V(z, \tau)| d z d \tau \leq C N_{h}(V) .
\end{aligned}
$$

An induction yields

$$
\begin{aligned}
\int_{D}\left|J_{k}(x, t ; y, s)\right| d y & \leq \int_{D} \int_{s}^{t} \int_{D}\left|J_{k-1}(x, t ; z, \tau)\right| V(z, \tau) \mid G_{0}(z, \tau ; y, s) d z d \tau d y \\
& \leq C \int_{s}^{t} \int_{D}\left|J_{k-1}(x, t ; z, \tau)\right| V(z, \tau) \mid d z d \tau \\
& \leq C_{0}^{k-1} \int_{s}^{t} \int_{D} G_{0}(x, t ; z, \tau)|V(z, \tau)| d z d \tau\left(N_{h}(V)\right)^{k-1} \\
& \leq C_{0}^{k}\left(N_{h}(V)\right)^{k}
\end{aligned}
$$

where $k=1,2, \ldots$ and $C_{0}$ is a constant. Now from (3.1) we have

$$
\begin{aligned}
\int_{D} \mid & G(x, t ; y, s)\left|d y \leq \sum_{k=0}^{\infty} \int_{D}\right| J_{k}(x, t ; y, s) \mid d y \\
& \leq C+\int_{s}^{t} \int_{D} G_{0}(x, t ; z, \tau)|V(z, \tau)| d z d \tau \sum_{k=0}^{\infty} C_{0}^{k}\left(N_{h}(V)\right)^{k} \\
& \leq C+\sum_{k=1}^{\infty} C_{0}^{k}\left(N_{h}(V)\right)^{k} .
\end{aligned}
$$

When $C_{0} N_{h}(V)<1$,

$$
\int_{D}|G(x, t ; y, s)| d y \leq C .
$$

This finishes the proof of Proposition 3.1. q.e.d.

The remaining two propositions in the section deal with the first variables of the Green's function.

Following the above argument and replacing $N_{h}(V)$ by $N_{h}^{*}(V)$, we have

Proposition 3.2. There exists $h_{0}>0$ such that the following statements are true when $0<h \leq h_{0}$.

(a) $\|G(., . ; y, s)\|_{L^{p}\left(Q_{h, s}^{+}\right)} \leq C_{0}, \quad 1<p<(n+2) / n$.

(b) $\left\|\nabla_{x} G(., . ; y, s)\right\|_{L^{p}\left(Q_{h, s}^{+}\right)} \leq C_{1}, \quad 1<p<(n+2) /(n+1)$.

(c) $\int_{D}|G(x, t ; y, s)| d x \leq C_{3}$, when $0<t-s<h_{0}$.

Here $Q_{h, s}^{+} \equiv D \times[s, s+h]$ and $h_{0}, C_{0}, C_{1}$ and $C_{2}$ depend only on $G_{0}$ and on the rate of convergence of $N_{h}^{*}(V)$ to zero when $h \rightarrow 0$.

In the next proposition we will extend the estimates in Proposition 3.2 to $D \times$ $[0, T]$. 
Proposition 3.3. Let $G$ be the Green's function of (1.1). Suppose that $V \in$ $L^{\infty}(D \times[0, T])$. There exists a constant $C$, depending on the rate of convergence of $N_{h}(V)+N_{h}^{*}(V)$ to zero when $h \rightarrow 0$ but not directly on $\|V\|_{L^{\infty}}$, such that

(a) $\|G(., . ; y, s)\|_{L^{p}(D \times[s, T])} \leq C, \quad 1<p<(n+2) / n$;

(b) $\left\|\nabla_{x} G(., . ; y, s)\right\|_{L^{p}(D \times[s, T])} \leq C$,

here $p<(n+2) /(n+1)$ and $(y, s) \in D \times[0, T]$;

(c) $\int_{D}|G(x, t ; y, s)| d x \leq C, \quad t>s$.

Proof. We will only give a proof of (b). Statements (a) and (c) can be treated similarly. Fixing any $(y, s) \in D \times[0, T]$ we subdivide $[s, T]$ into intervals with length not greater than $h_{0}$. The dividing points are

$$
s=s_{0}<s_{1}<\ldots<s_{m}
$$

with $s_{i}=s+i h_{0}$ and $T \in\left[s_{m-1}, s_{m}\right]$.

Then

$$
\left\|\nabla_{x} G(., . ; y, s)\right\|_{L^{p}(D \times[s, T])}^{p} \leq \sum_{i=1}^{m}\left\|\nabla_{x} G(., . ; y, s)\right\|_{L^{p}\left(D \times\left[s_{i-1}, s_{i}\right]\right)}^{p} .
$$

When $(x, t) \in D \times\left[s_{i-1}, s_{i}\right]$, using the reproducing property of $G$,

$$
\begin{array}{r}
G(x, t ; y, s)=\int_{D} \ldots \int_{D} G\left(x, t ; z_{i}, s_{i-1}\right) G\left(z_{i}, s_{i-1} ; z_{i-1}, s_{i-2}\right) \\
\ldots G\left(z_{1}, s_{1} ; y, s\right) d z_{1} d z_{2} \ldots d z_{i} .
\end{array}
$$

Hence for $\phi \in C^{\infty}\left(D \times\left[s_{i-1}, s_{i}\right]\right)$

$$
\begin{aligned}
& \left|\int_{s_{i-1}}^{s_{i}} \int_{D} \nabla_{x} G(x, t ; y, s) \phi(x, t) d x d t\right| \\
& \leq \int_{D} \ldots \int_{D} \int_{s_{i-1}}^{s_{i}} \int_{D}\left|\nabla_{x} G\left(x, t ; z_{i}, s_{i-1}\right) \phi(x, t)\right| d x d t G\left(z_{i}, s_{i-1} ; z_{i-1}, s_{i-2}\right) \\
& \ldots G\left(z_{1}, s_{1} ; y, s\right) d z_{1} d z_{2} \ldots d z_{i} \\
& \leq C_{1}\|\phi\|_{L^{p^{\prime}}\left(D \times\left[s_{i-1}, s_{i}\right]\right)} \int_{D} \ldots \int_{D} G\left(z_{i}, s_{i-1} ; z_{i-1}, s_{i-2}\right) \ldots G\left(z_{1}, s_{1} ; y, s\right) d z_{1} d z_{2} \ldots d z_{i} \\
& \leq C_{0}^{i} C_{1}\|\phi\|_{L^{p^{\prime}}\left(D \times\left[s_{i-1}, s_{i}\right]\right)} .
\end{aligned}
$$

Here the last two inequalities are derived from Proposition 3.2. It follows that

$$
\left\|\nabla_{x} G(., . ; y, s)\right\|_{L^{p}\left(D \times\left[s_{i-1}, s_{i}\right]\right)} \leq C_{0}^{i} C_{1},
$$

which implies, through (3.10),

$$
\left\|\nabla_{x} G(., . ; y, s)\right\|_{L^{p}(D \times[s, T])}^{p} \leq \sum_{i=1}^{m}\left(C_{0}^{i} C_{1}\right)^{p} \equiv C_{2}^{p} .
$$

Here $C_{2}$ depends only on $h_{0}, T$, and $G_{0}$. This proves the proposition. q.e.d.

In the remainder of the section we will consider some comparison properties of the Green's function. We will use $G^{\prime}$ to denote the Green's function of equation (1.1) with $V$ replaced by another function $U$. Unless stated otherwise, all the constants $C$ depend on the rate of convergence of $N_{h}(V)+N_{h}^{*}(V)$ when $h \rightarrow 0$, but they do not depend on $\|V\|_{L^{\infty}}$. 
Lemma 3.4. There are positive constants $C, h$ such that

$$
\begin{aligned}
& \left\|G^{\prime}(., . ; y, s)-G(., . ; y, s)\right\|_{L^{p}(D \times[s, T])} \leq C N_{h}^{*}(U-V), \\
& \left\|\nabla_{x} G^{\prime}(., . ; y, s)-\nabla_{x} G(., . ; y, s)\right\|_{L^{p}(D \times[s, T])} \leq C N_{h}^{*}(U-V),
\end{aligned}
$$

when $0<p<(n+2) /(n+1)$.

Proof. We will only prove the first inequality. The second one can be proven similarly. By (3.1) we know that $G$ and $G^{\prime}$ respectively satisfy the next two relations:

$$
\begin{gathered}
G=G_{0}-G *\left(V G_{0}\right), \\
G^{\prime}=G_{0}-G^{\prime} *\left(U G_{0}\right) .
\end{gathered}
$$

Hence

$$
G^{\prime}-G=G *\left[(V-U) G_{0}\right]-\left(G^{\prime}-G\right) *\left(U G_{0}\right) \equiv F-\left(G^{\prime}-G\right) *\left(U G_{0}\right)
$$

and formally we have that

$$
G^{\prime}-G=\sum_{k=0}^{\infty}(-1)^{k} F *\left(U G_{0}\right)^{* k}
$$

Following the derivation of (3.6) in Proposition 3.1, we obtain

$$
\begin{aligned}
& \left\|G^{\prime}(., . ; y, s)-G(., . ; y, s)\right\|_{L^{p}(D \times[s, T])} \\
& \quad \leq C \sum_{k=0}^{\infty}\left[N_{h}^{*}(U)\right]^{k} \sup _{(z, \tau)}\|F(., . ; z, \tau)\|_{L^{p}(D \times[\tau, T])},
\end{aligned}
$$

where $p$ is a number larger than $1, \tau \geq s$ and $0<T-s \leq h$. Since $U$ is in the parabolic Kato class, the right-hand side of (3.13) is convergent when $h$ is sufficiently small.

To estimate $\|F\|_{L^{p}}$ let us recall from (3.11) that

$$
F \equiv F(x, t ; z, \tau)=\int_{\tau}^{t} \int_{D} G(x, t ; w, l)[V(w, l)-U(w, l)] G_{0}(w, l ; z, \tau) d w d l .
$$

Given $\phi \in C^{\infty}(D \times[\tau, T])$, we then have

$$
\begin{aligned}
& \left|\int_{\tau}^{T} \int_{D} F(x, t ; z, \tau) \phi(x, t) d x d t\right| \\
& \leq \int_{\tau}^{T} \int_{D} \int_{\tau}^{t} \int_{D}\left|G(x, t ; w, l)[V(w, l)-U(w, l)] G_{0}(w, l ; z, \tau) \phi(x, t)\right| d w d l d x d t \\
& =\int_{\tau}^{T} \int_{D} \int_{l}^{T} \int_{D}|G(x, t ; w, l) \phi(x, t)| d x d t|V(w, l)-U(w, l)| G_{0}(w, l ; z, \tau) d w d l \\
& \leq C \sup _{w, l}\|G(., . ; w, l)\|_{L^{p}(D \times[l, T])}\|\phi\|_{L^{p^{\prime}(D \times[l, T])}} N_{h}^{*}(V-U) .
\end{aligned}
$$


So by Proposition 3.2

$$
\|F(., . ; z, \tau)\|_{L^{p}(D \times[\tau, T])} \leq C N_{h}^{*}(V-U),
$$

when $h$ is sufficiently small. Coupling (3.13) and (3.14) we obtain

$\left\|G^{\prime}(., . ; y, s)-G(., . ; y, s)\right\|_{L^{p}(D \times[s, T])} \leq C \sum_{k=0}^{\infty}\left[N_{h}^{*}(U)\right]^{k} N_{h}^{*}(V-U) \leq C N_{h}^{*}(V-U)$,

when $T-s \leq h$.

In case $T-s>h$ we take a partition of $[s, T]$ as

$$
s=s_{0}<s_{1}<\ldots<s_{m}=T,
$$

where $s_{j+1}-s_{j} \leq h$ for $j=1,2, \ldots, m-1$. The lemma will be proven if we can show that

$$
\left\|G^{\prime}(., . ; y, s)-G(., . ; y, s)\right\|_{L^{p}\left(D \times\left[s_{j}, s_{j+1}\right]\right)} \leq C N_{h}^{*}(V-U)
$$

for each $j=1, \ldots, m$. To this end let us choose $t \in\left[s_{j}, s_{j+1}\right]$. By the reproducing property of the Green's functions we obtain

$$
\begin{aligned}
& G^{\prime}(x, t ; y, s)-G(x, t ; y, s) \\
& =\int_{D}\left[G^{\prime}\left(x, t ; z, s_{j}\right) G^{\prime}\left(z, s_{j} ; y, s\right)-G\left(x, t ; z, s_{j}\right) G\left(z, s_{j} ; y, s\right)\right] d z \\
& =\int_{D}\left[G^{\prime}\left(x, t ; z, s_{j}\right)-G\left(x, t ; z, s_{j}\right)\right] G^{\prime}\left(z, s_{j} ; y, s\right) d z \\
& \quad+\int_{D} G\left(x, t ; z, s_{j}\right)\left[G^{\prime}\left(z, s_{j} ; y, s\right)-G\left(z, s_{j} ; y, s\right)\right] d z .
\end{aligned}
$$

Hence we have, for any $\phi \in C\left(D \times\left[s_{j}, s_{j+1}\right]\right),\|\phi\|_{L^{p^{\prime}}\left(D \times\left[s_{j}, s_{j+1}\right]\right)} \leq 1$,

$$
\begin{aligned}
& \quad\left|\int_{s_{j}}^{s_{j+1}} \int_{D}\left[G^{\prime}(x, t ; y, s)-G(x, t ; y, s)\right] \phi(x, t) d x d t\right| \\
& \leq \int_{D} \int_{s_{j}}^{s_{j+1}} \int_{D}\left|\left[G^{\prime}\left(x, t ; z, s_{j}\right)-G\left(x, t ; z, s_{j}\right)\right] \phi(x, t)\right| d x d t G^{\prime}\left(z, s_{j} ; y, s\right) d z \\
& \quad+\int_{D} \int_{s_{j}}^{s_{j+1}} \int_{D}\left|G\left(x, t ; z, s_{j}\right) \phi(x, t)\right| d x d t\left|G^{\prime}\left(z, s_{j} ; y, s\right)-G\left(z, s_{j} ; y, s\right)\right| d z \\
& \leq C N_{h}^{*}(U-V) \int_{D} G^{\prime}\left(z, s_{j} ; y, s\right) d z+C \int_{D}\left|G^{\prime}\left(z, s_{j} ; y, s\right)-G\left(z, s_{j} ; y, s\right)\right| d z
\end{aligned}
$$

where we used (3.14') and Proposition 3.2. Finally with the help of Proposition 3.3 and the next lemma we have that

$$
\left|\int_{s_{j}}^{s_{j+1}} \int_{D}\left[G^{\prime}(x, t ; y, s)-G(x, t ; y, s)\right] \phi(x, t) d x d t\right| \leq C N_{h}^{*}(U-V),
$$

which is (3.15). q.e.d. 
Lemma 3.5. There are positive constants $C$ and $h$ such that

$$
\begin{aligned}
& \int_{D}\left|G^{\prime}(x, t ; y, s)-G(x, t ; y, s)\right| d y \leq C N_{h}(U-V), \\
& \int_{D}\left|G^{\prime}(x, t ; y, s)-G(x, t ; y, s)\right| d x \leq C N_{h}^{*}(U-V) .
\end{aligned}
$$

In particular, if we take $U=0$ then

$$
\int_{D}|G(x, t ; y, s)| d y \leq C N_{h}(V) .
$$

Proof. Let us prove the first statement.

Step 1. By (3.11)

$$
\begin{aligned}
& G^{\prime}(x, t ; y, s)-G(x, t ; y, s) \\
& \quad=F(x, t ; y, s)-\int_{s}^{t} \int_{D}\left[G^{\prime}(x, t ; z, \tau)-G(x, t ; z, \tau)\right] U(z, \tau) G_{0}(z, \tau ; y, s) d z d \tau
\end{aligned}
$$

and so

(3.16)

$$
\begin{aligned}
& \int_{D}\left|G^{\prime}(x, t ; y, s)-G(x, t ; y, s)\right| d y \leq \int_{D}|F(x, t ; y, s)| d y \\
& \quad+\int_{s}^{t} \int_{D}\left|G^{\prime}(x, t ; z, \tau)-G(x, t ; z, \tau)\right||U(z, \tau)| \int_{D}\left|G_{0}(z, \tau ; y, s)\right| d y d z d \tau \\
& \leq \int_{D}|F(x, t ; y, s)| d y+C \int_{s}^{t} \int_{D}\left|G^{\prime}(x, t ; z, \tau)-G(x, t ; z, \tau)\right||U(z, \tau)| d z d \tau \\
& \leq \int_{D}|F(x, t ; y, s)| d y+C \sum_{k=0}^{\infty} \int_{s}^{t} \int_{D}\left|F *\left(U G_{0}\right)^{* k}(x, t ; z, \tau) U(z, \tau)\right| d z d \tau
\end{aligned}
$$

by (3.12).

To control the last two terms of (3.16) we observe that

$$
\begin{aligned}
\int_{D}|F(x, t ; y, s)| d y \\
\quad \leq \int_{s}^{t} \int_{D}|G(x, t ; z, \tau)[V(z, \tau)-U(z, \tau)]| \int_{D}\left|G_{0}(z, \tau ; y, s)\right| d y d z d \tau \\
\quad \leq C \int_{s}^{t} \int_{D}|G(x, t ; z, \tau)[V(z, \tau)-U(z, \tau)]| d z d \tau \\
\quad \leq C \sum_{k=0}^{\infty} \int_{s}^{t} \int_{D}\left|G_{0} *\left(V G_{0}\right)^{* k}(x, t ; z, \tau)[V(z, \tau)-U(z, \tau)]\right| d z d \tau
\end{aligned}
$$

Following the argument in Proposition 3.1 we obtain, for a $C_{1}>0$, that

$$
\int_{D}|F(x, t ; y, s)| d y \leq C \sum_{k=0}^{\infty}\left[C_{1}\left(N_{h}(V)+N_{h}^{*}(V)\right)\right]^{k} N_{h}(V-U),
$$


whenever $0<t-s<h$. Next, since

$$
\begin{aligned}
& \int_{s}^{t} \int_{D}\left|F *\left(U G_{0}\right)^{* k}(x, t ; z, \tau) U(z, \tau)\right| d z d \tau \\
& \leq \int_{s}^{t} \int_{D} \int_{\tau}^{t} \int_{D}\left|F *\left(U G_{0}\right)^{* k-1}(x, t ; w, l) U(w, l) G_{0}(w, l ; z, \tau) U(z, \tau)\right| d w d l d z d \tau \\
& =\int_{s}^{t} \int_{D}\left|F *\left(U G_{0}\right)^{* k-1}(x, t ; w, l) U(w, l)\right| \int_{s}^{l} \int_{D}\left|G_{0}(w, l ; z, \tau) U(z, \tau)\right| d z d \tau d w d l \\
& \leq C_{0} \int_{s}^{t} \int_{D}\left|F *\left(U G_{0}\right)^{* k-1}(x, t ; w, l) U(w, l)\right| d w d l N_{h}(U)
\end{aligned}
$$

we can deduce, by an induction, that

$$
\begin{aligned}
& \int_{s}^{t} \int_{D}\left|F *\left(U G_{0}\right)^{* k}(x, t ; z, \tau) U(z, \tau)\right| d z d \tau \\
& \quad \leq C C_{0}^{k}\left(N_{h}(U)\right)^{k} \int_{s}^{t} \int_{D}|F(x, t ; z, \tau) U(z, \tau)| d z d \tau \\
& \quad \leq C C_{0}^{k}\left(N_{h}(U)\right)^{k} \int_{s}^{t} \int_{D} \int_{\tau}^{t} \int_{D}\{\mid G(x, t ; w, l)[V(w, l)-U(w, l)] \\
& \quad \leq C C_{0}^{k}\left(N_{h}(U)\right)^{k} \int_{s}^{t} \int_{D}\{|G(x, t ; w, l)[V(w, l)-U(w, l)]| \\
& \quad \leq C C_{0}^{k}\left(N_{h}(U)\right)^{k+1} \int_{s}^{t} \int_{D}|G(x, t ; w, l)[V(w, l)-U(w, l)]| d w d l \\
& \left.\left.\quad \leq C C_{0}^{k}\left(N_{h}(U)\right)^{k+1} \sum_{j=0}^{\infty} C_{1}^{j}\left(N_{h}(V)+G_{0}(w, l ; z, \tau) U(z, \tau) \mid\right\} d z d \tau d w d l\right]\right\} d w d l d z d \tau \\
& \quad \leq C C_{0}^{k}\left(N_{h}(U)\right)^{k+1} N_{h}(V-U),
\end{aligned}
$$

when $0<t-s \leq h$, which is sufficiently small. Note that in order to go from the third from last to the second from last term in (3.18) we used the fact that

$$
\begin{aligned}
& \int_{s}^{t} \int_{D}|G(x, t ; w, l)[V(w, l)-U(w, l)]| d w d l \\
& \leq \int_{s}^{t} \int_{D} \sum_{j=0}^{\infty}\left|G_{0} *\left(-V G_{0}\right)^{* j}(x, t ; w, l)[V(w, l)-U(w, l)]\right| d w d l \\
& \leq C \sum_{j=0}^{\infty} C_{1}^{j}\left(N_{h}(V)+N_{h}^{*}(V)\right)^{j} N_{h}(V-U) \quad \text { (by the method in Proposition 3.1) } \\
& \leq C N_{h}(V-U) \text { when } \quad 0<t-s \leq h .
\end{aligned}
$$

Combining (3.16), (3.17) and (3.18) we have

$$
\int_{D}\left|G^{\prime}(x, t ; y, s)-G(x, t ; y, s)\right| d y \leq C N_{h}(V-U),
$$

when $0<t-s \leq h$. 
Step 2. In case $t-s>h$ we take a partition of $[s, t]$ as

$$
s=s_{0}<s_{1}<\ldots<s_{m}=t,
$$

where $s_{j+1}-s_{j} \leq h$ for $j=0, \ldots, m-1$. Using the reproducing property of the Green's functions and denoting $x$ by $z_{m}$ and $y$ by $z_{0}$, we have

$$
\begin{aligned}
& \int_{D}\left|G^{\prime}(x, t ; y, s)-G(x, t ; y, s)\right| d y \\
& \leq \int_{D} \int_{D} \ldots \int_{D}\left\{\mid G^{\prime}\left(x, t ; z_{m-1}, s_{m-1}\right) \ldots G^{\prime}\left(z_{1}, s_{1} ; y, s\right)\right. \\
& \left.\quad-G\left(x, t ; z_{m-1}, s_{m-1}\right) \ldots G\left(z_{1}, s_{1} ; y, s\right) \mid\right\} d z_{1} \ldots d z_{m-1} d y \\
& \leq \sum_{j=1}^{m} \int_{D} \int_{D} \ldots \int_{D} \prod_{l=j+1}^{m} G\left(z_{l}, s_{l} ; z_{l-1}, s_{l-1}\right)\left|\left(G^{\prime}-G\right)\left(z_{j}, s_{j} ; z_{j-1}, s_{j-1}\right)\right| \\
& \quad \times \prod_{l=1}^{j-1} G^{\prime}\left(z_{l}, s_{l} ; z_{l-1}, s_{l-1}\right) d y d z_{1} \ldots d z_{m-1} \\
& \leq \sum_{j=1}^{m} C^{j-1} \int_{D} \ldots \int_{D}\left\{\prod_{l=j+1}^{m} G\left(z_{l}, s_{l} ; z_{l-1}, s_{l-1}\right)\right. \\
& \left.\quad \times \int_{D}\left|\left(G^{\prime}-G\right)\left(z_{j}, s_{j} ; z_{j-1}, s_{j-1}\right)\right| d z_{j-1}\right\} d z_{j} \ldots d z_{m-1} \\
& \leq \sum_{j=1}^{m} C^{j-1} \int_{D} \ldots \int_{D} \prod_{l=j+1}^{m} G\left(z_{l}, s_{l} ; z_{l-1}, s_{l-1}\right) d z_{j} \ldots d z_{m-1} C N_{h}(U-V) \\
& \leq C N_{h}(U-V) .
\end{aligned}
$$

Similarly we have

$$
\int_{D}\left|G^{\prime}(x, t ; y, s)-G(x, t ; y, s)\right| d x \leq C N_{h}^{*}(U-V) .
$$

This concludes the proof. q.e.d.

We conclude this section with a corollary about the case when $V$ is not an $L^{\infty}$ function.

Corollary 3.6. Suppose that $V$ is in the parabolic Kato class. Then there exists $h_{0}>0$ such that the following statement is true.

When $0<t-s \leq h_{0}$ the function $G(x, t ; y, s)$ given by (3.1) is well defined in the sense that

(a) $G(., . ; y, s) \in L^{p}\left(s, s+h_{0} ; W^{1, p}(D)\right)$ for a $p>1$;

(b) $G(x, t ; .,.) \in L^{p}\left(t-h_{0}, t ; W^{1, p}(D)\right)$ for a $p>1$;

(c) $G(., t ; y, s) \in L^{1}(D)$ and $G(x, t ; ., s) \in L^{1}(D)$. 
Moreover, all conclusions of Proposition 3.1, Proposition 3.2, Proposition 3.3, Lemma 3.4 and Lemma 3.5 still hold for $G$.

Proof. Going through the argument of Proposition 3.1, Proposition 3.2, Proposition 3.3, Lemma 3.4 and Lemma 3.5, one finds that the boundedness of $V$ is irrelevant when $0<t-s \leq h_{0}$. Hence the corollary is true. q.e.d.

\section{Proof of Theorem A: The initial-Dirichlet problem}

\section{Proof of Theorem A.}

Step 1. Construction of the Green's function. For any positive integer $k$ define

$$
V_{k}(x, t)= \begin{cases}-k, & \text { if } V(x, t) \leq-k \\ V(x, t), & \text { if }-k \leq V(x, t) \leq k ; \\ k, & \text { if } V(x, t) \geq k\end{cases}
$$

Thus $V_{k}$ is an $L^{\infty}$ function and $\lim _{k \rightarrow \infty} V_{k}(x)=V(x)$ a.e. Moreover

$$
\begin{aligned}
& N_{h}\left(V_{k}\right)+N_{h}^{*}\left(V_{k}\right) \leq N_{h}(V)+N_{h}^{*}(V), \\
& N_{h}\left(V_{k}-V\right)+N_{h}^{*}\left(V_{k}-V\right) \rightarrow 0, \quad \text { when } \quad k \rightarrow \infty
\end{aligned}
$$

for any $h>0$.

By the standard theory we know the existence and uniqueness for the Green's function $G_{k}(x, t ; y, s)$ of the operator $H_{k}$ defined by

$$
H_{k} u(x, t) \equiv \sum_{i, j=1}^{n} \frac{\partial}{\partial x_{i}}\left(a_{i j}(x, t) \frac{\partial u}{\partial x_{j}}\right)(x, t)-V_{k}(x, t) u(x, t)-\frac{\partial u}{\partial t}(x, t)=0 .
$$

In view of Lemma 3.4, for a $p>1$ and $h>0$ and positive integers $k$ and $k_{1}$,

$$
\left\|G_{k}(., . ; y, s)-G_{k_{1}}(., . ; y, s)\right\|_{L^{p}\left(s, T ; W^{1, p}(D)\right)} \leq C N_{h}^{*}\left(V_{k}-V_{k_{1}}\right) .
$$

Hence the sequence $\left\{G_{k}(., ; y, s)\right\}$ is convergent in $L^{p}\left(s, T ; W^{1, p}(D)\right)$ norm for a $p>1$. Moreover there is a constant $C$ independent of $k$ and $p$ such that

$$
\left\|G_{k}(., . ; y, s)\right\|_{L^{p}\left(s, T ; W^{1, p}(D)\right)} \leq C .
$$

Definition. Our candidate $G(x, t ; y, s)$ for the Green's function of $(1.1)$ is defined in the following way.

(i). Let $h_{0}$ be the positive number in Corollary 3.6. When $0<t-s \leq h_{0}$,

$$
G(., . ; y, s) \equiv \lim _{k \rightarrow \infty} G_{k}(., . ; y, s) \quad \text { in } \quad L^{p}\left(s, s+h_{0} ; W^{1, p}(D)\right) .
$$

Note that the limit exists by Corollary 3.6.

(ii). When $t \in\left(s+j h_{0}, s+(j+1) h_{0}\right]$ for a positive integer $j$,

$$
G(x, t ; y, s) \equiv \int_{D} \ldots \int_{D} G\left(x, t ; z_{j}, s+j h_{0}\right) \ldots G\left(z_{1}, s+h_{0} ; y, s\right) d z_{j} \ldots d z_{1} .
$$


By Corollary 3.6, in the case $0<t-s \leq h_{0}, G(x, t ; y, s)$ is explicitly given by (3.1), which means $G=\sum_{k=0}^{\infty} G_{0} *\left(-V G_{0}\right)^{* k}$. We also need some explanation about the integrals in (ii).

Claim. The integrals are well defined for for a.e $x$ and a.e. $y$ with respect to $n$ dimensional Lebesgue measure respectively.

Proof of the claim. Without any loss of generality let us show that the claim is true for $G(x, t ; y, s) \equiv \int_{D} G\left(x, t ; z, s+h_{0}\right) G\left(z, s+h_{0} ; y, s\right) d z$, where $t \leq s+2 h_{0}$. Obviously Corollary 3.6 is applicable to $G\left(x, t ; z, s+h_{0}\right)$ and $G\left(z, s+h_{0} ; y, s\right)$. Hence by Fubini's theorem

$$
\begin{aligned}
& \int_{D} \int_{D} G\left(x, t ; z, s+h_{0}\right) G\left(z, s+h_{0} ; y, s\right) d z d x \leq C, \\
& \int_{D} \int_{D} G\left(x, t ; z, s+h_{0}\right) G\left(z, s+h_{0} ; y, s\right) d z d y \leq C .
\end{aligned}
$$

Therefore we know that the claim is true by Fubini's theorem once again. This proves the claim.

In view of Corollary 3.6 and the last claim, we know that $G$ thus defined satisfies all the properties given by Propositions 3.1, 3.2, 3.3 and Lemmas 3.4, 3.5. In fact all arguments in the last section can be made for $G$ without a change. So when we use these propositions and lemmas from section 3, we will include $G$. For convenience we list some of the properties:

$$
\left\{\begin{array}{l}
\left\|G_{k}(., . ; y, s)-G(., . ; y, s)\right\|_{L^{p}\left(s, T ; W^{1, p}(D)\right)} \leq C N_{h}^{*}\left(V_{k}-V\right), \quad h>0 \\
\int_{D}\left|G_{k}(x, t ; y, s)-G(x, t ; y, s)\right| d y \leq C N_{h}\left(V_{k}-V\right) \quad(\text { from Lemma 3.5) }
\end{array}\right.
$$

We are going to show that $G$ is indeed the Green's function of the operator in (1.1). To this end we need to show, for a function $f \in C_{0}^{\infty}(D)$, that the function

$$
u(x, t) \equiv \int_{D} G(x, t ; y, s) f(y) d y
$$

satisfies

(i). $u$ is a weak solution of (1.1). This means, for any $\phi \in C_{0}^{\infty}(D \times[s, T])$,

$$
\begin{aligned}
\int_{s}^{T} \int_{D}\left\{-\sum_{i, j=1}^{n} a_{i j}(x, t)\right. & \frac{\partial}{\partial x_{j}} u(x, t) \frac{\partial}{\partial x_{i}} \phi(x, t) \\
& \left.-V(x, t) u(x, t) \phi(x, t)+u(x, t) \frac{\partial \phi}{\partial t}(x, t)\right\} d x d t=0 .
\end{aligned}
$$

(ii).

$$
\lim _{t \rightarrow s^{+}} u(x, t)=f(x)
$$

(iii). Given $\left(x_{0}, t_{0}\right) \in \partial D \times(s, T]$ and $\left\{\left(x_{m}, t_{m}\right)\right\} \subset D \times(s, T]$, which converges to $\left(x_{0}, t_{0}\right)$ when $m \rightarrow \infty$,

$$
\lim _{m \rightarrow \infty} u\left(x_{m}, t_{m}\right)=0 .
$$


(iv).

$$
u \in C^{0}\left([0, T] ; L^{2}(D)\right) \cap L^{2}\left(0, T ; W_{0}^{1,2}(D)\right) .
$$

The proof of the statements above is divided into several steps.

Step 2. Proof of (4.2). Let us verify (4.2) first. By the standard theory in $[\mathrm{A}]$, the function

$$
u_{k}(x, t) \equiv \int_{D} G_{k}(x, t ; y, s) f(y) d y
$$

is a weak solution of $H_{k}$ and hence satisfies

$$
\begin{gathered}
-\int_{s}^{T} \int_{D} \sum_{i, j=1}^{n} a_{i j} \frac{\partial}{\partial x_{j}} u_{k}(x, t) \frac{\partial}{\partial x_{i}} \phi(x, t) d x d t-\int_{s}^{T} \int_{D} V_{k}(x, t) u_{k}(x, t) \phi(x, t) d x d t \\
+\int_{s}^{T} \int_{D} u_{k}(x, t) \frac{\partial \phi}{\partial t}(x, t) d x d t \equiv-A_{k}-B_{k}+C_{k}=0 .
\end{gathered}
$$

Given a function $\eta \in C_{0}^{\infty}(D \times[s, T])$, we have

$$
\begin{aligned}
\mid \int_{s}^{T} & \int_{D} u_{k}(x, t) \nabla_{x} \eta(x, t) d x d t \mid \\
& =\left|\int_{s}^{T} \int_{D} \int_{D} G_{k}(x, t ; y, s) f(y) \nabla_{x} \eta(x, t) d y d x d t\right| \\
& =\left|\int_{D} f(y) \int_{s}^{T} \int_{D} G_{k}(x, t ; y, s) \nabla_{x} \eta(x, t) d x d t d y\right| \\
& =\left|\int_{D} f(y) \int_{s}^{T} \int_{D} \nabla_{x} G_{k}(x, t ; y, s) \eta(x, t) d x d t d y\right| \\
& \leq\|f\|_{L^{1}(D)} \sup _{y, s}\left\|\nabla_{x} G_{k}(., . ; y, s)\right\|_{L^{p}(D \times[s, T])}|| \eta \|_{L^{p^{\prime}}(D \times[s, T])} \\
& \leq C\|f\|_{L^{1}(D)}\|\eta\|_{L^{p^{\prime}}(D \times[s, T])}, p<\frac{n+2}{n+1}, \quad \text { by Proposition 3.3. }
\end{aligned}
$$

Hence there is a constant $C$ independent of $k$ such that

$$
\left\|\nabla_{x} u_{k}\right\|_{L^{p}(D \times[s, T])} \leq C .
$$

On the other hand

$$
\begin{aligned}
\left|u_{k}(x, t)-u(x, t)\right| & =\left|\int_{D}\left(G_{k}(x, t ; y, s)-G(x, t ; y, s)\right) f(y) d y\right| \\
& \leq C \int_{D}\left|G_{k}(x, t ; y, s)-G(x, t ; y, s)\right| d y \\
& \leq C N_{h}\left(V_{k}-V\right) \quad \text { (by Lemma 3.5). }
\end{aligned}
$$

Hence

$$
\lim _{k \rightarrow \infty} u_{k}(x, t)=u(x, t) \quad \text { uniformly. }
$$


In view of (4.5) and (4.6) we can find a subsequence of $\left\{u_{k}\right\}$, which is still called $\left\{u_{k}\right\}$, such that

$$
\lim _{k \rightarrow \infty} \nabla_{x} u_{k}=\nabla_{x} u, \quad \text { weakly in } L^{p} .
$$

This immediately tells us that

$$
\lim _{k \rightarrow \infty} A_{k}=-\int_{s}^{T} \int_{D} \sum_{i, j=1}^{n} a_{i j}(x, t) \frac{\partial}{\partial x_{j}} u(x, t) \frac{\partial}{\partial x_{i}} \phi(x, t) d x d t .
$$

Next we notice that

$$
\begin{aligned}
B_{k} & =\int_{s}^{T} \int_{D} V_{k}(x, t) u_{k}(x, t) \phi(x, t) d x d t \\
& =\int_{s}^{T} \int_{D} \int_{D} V_{k}(x, t) G_{k}(x, t ; y, s) f(y) \phi(x, t) d y d x d t
\end{aligned}
$$

and

$$
\begin{aligned}
B & \equiv \int_{s}^{T} \int_{D} V(x, t) u(x, t) \phi(x, t) d x d t \\
& =\int_{s}^{T} \int_{D} \int_{D} V(x, t) G(x, t ; y, s) f(y) \phi(x, t) d y d x d t .
\end{aligned}
$$

Hence

$$
\begin{aligned}
& \left|B_{k}-B\right| \\
& \leq \int_{s}^{T} \int_{D} \int_{D}\left|V_{k}(x, t) G_{k}(x, t ; y, s)-V(x, t) G(x, t ; y, s)\right||f(y) \phi(x, t)| d y d x d t \\
& \leq C \int_{s}^{T} \int_{D} \int_{D}\left|V_{k}(x, t) G_{k}(x, t ; y, s)-V(x, t) G(x, t ; y, s)\right| d y d x d t \\
& \leq C \int_{s}^{T} \int_{D}\left|V_{k}(x, t)-V(x, t)\right| \int_{D} G_{k}(x, t ; y, s) d y d x d t \\
& \quad+C \int_{s}^{T} \int_{D}|V(x, t)| \int_{D}\left|G_{k}(x, t ; y, s)-G(x, t ; y, s)\right| d y d x d t \\
& \leq C \int_{s}^{T} \int_{D}\left|V_{k}(x, t)-V(x, t)\right| d x d t+C \int_{s}^{T} \int_{D}|V(x, t)| d x d t N_{h}\left(V_{k}-V\right) .
\end{aligned}
$$

Here the last inequality is obtained by using Lemma 3.5 with an adequate positive number $h$.

Now it is clear that

$$
\lim _{k \rightarrow \infty} B_{k}=\int_{s}^{T} \int_{D} V(x, t) u(x, t) \phi(x, t) d x d t .
$$

Finally, it is easy to see that

$$
\lim _{k \rightarrow \infty} C_{k}=\int_{s}^{T} \int_{D} u(x, t) \frac{\partial \phi}{\partial t}(x, t) d x d t .
$$


Substituting (4.7), (4.8) and (4.9) into (4.4), we deduce (4.2).

Step 3. Proof of (4.3). Next we will show that (4.3) is valid. When $0<$ $t-s<h_{0}$, clearly we have the following expansion (see (3.1)):

$$
\begin{aligned}
& G(x, t ; y, s) \\
& \quad=G_{0}(x, t ; y, s)+G_{0} * H G_{0}(x, t ; y, s)+\ldots+G_{0} *\left(H G_{0}\right)^{* k}(x, t ; y, s)+\ldots ; \\
& \quad \equiv G_{0}(x, t ; y, s)+J_{1}(x, t ; y, s)+\ldots+J_{k}(x, t ; y, s)+\ldots,
\end{aligned}
$$

where $H G_{0}(x, t ; y, s) \equiv H_{x, t} G_{0}(x, t ; y, s)=-V(x, t) G_{0}(x, t ; y, s)$.

Since $G_{0}$ is the Green's function of $H_{0}$ we have

$$
\lim _{t \rightarrow s} \int_{D} G_{0}(x, t ; y, s) f(y) d y=f(x) .
$$

Next,

$$
\begin{aligned}
\left|\int_{D} J_{1}(x, t ; y, s) f(y) d y\right| & =\left|\int_{D} \int_{s}^{t} \int_{D} G_{0}(x, t ; z, \tau) V(z, \tau) G_{0}(z, \tau ; y, s) f(y) d z d \tau d y\right| \\
& \leq \int_{s}^{t} \int_{D} G_{0}(x, t ; z, \tau) V(z, \tau) \int_{D} G_{0}(z, \tau ; y, s)|f(y)| d y d z d \tau \\
& \leq C \sup _{D}|f| \int_{s}^{t} \int_{D} G_{0}(x, t ; z, \tau) V(z, \tau) d z d \tau \\
& \leq \sup _{D}|f| C N_{t-s}(V) .
\end{aligned}
$$

An easy induction yields

$$
\left|\int_{D} J_{k}(x, t ; y, s) f(y) d y\right| \leq \sup _{D}|f|\left[C N_{t-s}(V)\right]^{k} .
$$

Hence

$$
\left|\sum_{k=1}^{\infty} \int_{D} J_{k}(x, t ; y, s) f(y) d y\right| \leq \sup _{D}|f| \sum_{k=1}^{\infty}\left[C N_{t-s}(V)\right]^{k} .
$$

It follows that

$$
\lim _{t \rightarrow s} \sum_{k=1}^{\infty} \int_{D} J_{k}(x, t ; y, s) f(y) d y=0
$$

since $\lim _{t \rightarrow s} N_{t-s}(V)=0$.

Combining (4.10) with (4.11) and (4.13), we obtain

$$
\lim _{t \rightarrow s} \int_{D} G(x, t ; y, s) f(y) d y=\lim _{t \rightarrow s} \int_{D} G_{0}(x, t ; y, s) f(y) d y=f(x) .
$$

In fact, if only $f \in C_{0}(\bar{D})$, then (i), (ii) still hold.

Step 4. Proof of (iii). In view of (3.9') we have

$$
\begin{aligned}
& |u(x, t)| \leq \int_{D}|G(x, t ; y, s)||f(y)| d y \leq C \int_{D}|G(x, t ; y, s)| d y \\
& \leq C \int_{D}\left|G_{0}(x, t ; y, s)\right| d y+C \int_{s}^{t} \int_{D} G_{0}(x, t ; z, \tau)|V(z, \tau)| d z d \tau\left(\sum_{k=0}^{\infty} C_{0}^{k}\left(N_{h}(V)\right)^{k}\right)
\end{aligned}
$$


for all $t$ such that $0<t-s \leq h$. When $h$ is sufficiently small it follows that

$$
|u(x, t)| \leq C \int_{D}\left|G_{0}(x, t ; y, s)\right| d y+C \int_{s}^{t} \int_{D} G_{0}(x, t ; z, \tau)|V(z, \tau)| d z d \tau .
$$

According to Theorem 9 in $[\mathrm{A}], G_{0}(x, t ; z, \tau)$ is continuous as a function of $(x, t)$ in $\bar{D} \times(\tau, T]$ for each $(z, \tau) \in D \times(s, T]$. Hence for $\left(x_{0}, t_{0}\right) \in \partial D \times(s, T]$ and $\left\{\left(x_{m}, t_{m}\right)\right\} \subset D \times(s, T]$ such that $\lim _{m \rightarrow \infty}\left(x_{m}, t_{m}\right)=\left(x_{0}, t_{0}\right)$,

$$
\lim _{m \rightarrow \infty} \int_{D}\left|G_{0}\left(x_{m}, t_{m} ; y, s\right)\right| d y=0 .
$$

To control the last term of (4.14), recall that there is a constant $C$ such that

$$
G_{0}(x, t ; z, \tau) \leq \frac{C}{(t-\tau)^{n / 2}} \exp \left(-\alpha \frac{|x-z|^{2}}{t-\tau}\right) .
$$

Hence for any $\epsilon>0$, there is an $\eta>0$ such that, for all $(x, t) \in D \times(s, T]$,

$$
\int_{t-\eta}^{t} \int_{D} G_{0}(x, t ; z, \tau)|V(z, \tau)| d z d \tau<\epsilon / 2
$$

On the other hand, the dominated convergence theorem implies

$$
\int_{s}^{t_{m}-\eta} \int_{D} G_{0}\left(x_{m}, t_{m} ; z, \tau\right)|V(z, \tau)| d z d \tau<\epsilon / 2
$$

whenever $m$ is sufficiently large. But then we have

$$
\int_{s}^{t_{m}} \int_{D} G_{0}\left(x_{m}, t_{m} ; z, \tau\right)|V(z, \tau)| d z d \tau<\epsilon
$$

This shows, via (4.14), that

$$
\lim _{m \rightarrow \infty} u\left(x_{m}, t_{m}\right)=0
$$

for $t$ such that $0<t-s \leq h$, which is sufficiently small.

In the case when $t-s>h$ we can choose an $s_{1}>s$ such that $0<t-s_{1} \leq h$. Then by the reproducing formula of the Green's function we have that

$$
\begin{aligned}
|u(x, t)| & =\left|\int_{D} G(x, t ; y, s) f(y) d y\right| \\
& =\left|\int_{D} \int_{D} G\left(x, t ; z, s_{1}\right) G\left(z, s_{1} ; y, s\right) f(y) d z d y\right| \leq C \int_{D}\left|G\left(x, t ; z, s_{1}\right)\right| d z .
\end{aligned}
$$

The last term of the above inequality can then be treated in the same way as before.

Step 5. Proof of (iv). First we show that $u \in L^{2}\left(0, T ; W_{0}^{1,2}(D)\right)$. Recall from step 1 and step 2 that

$$
u(x, t)=\int_{D} G(x, t ; y, s) f(y) d y
$$


and

$$
u_{k}(x, t)=\int_{D} G_{k}(x, t ; y, s) f(y) d y .
$$

From Lemma 3.5 we know the existence of a constant $C$ independent of $k$ such that

$$
\left|u_{k}(x, t)\right| \leq\|f\|_{L^{\infty}(D)} \int_{D}\left|G_{k}(x, t ; y, s)\right| d y \leq C .
$$

Moreover $u_{k} \in L^{2}\left(0, T ; W_{0}^{1,2}(D)\right)$ is the weak solution of the following problem:

$$
\left\{\begin{array}{l}
\nabla\left(A \nabla u_{k}\right)-V_{k} u_{k}-\left(u_{k}\right)_{t}=0, \\
u_{k}(x, t)=0 \quad \text { when } \quad(x, t) \in \partial D \times[0, T], \\
u_{k}(x, 0)=f(x) .
\end{array}\right.
$$

Proceeding along the lines of $[\mathrm{A}]$ by choosing adequate test functions, we have

$$
\begin{aligned}
\int_{0}^{T} \int_{D}\left|\nabla u_{k}(x, t)\right|^{2} d x d t+\sup _{t \in[0, T]} \int_{D}\left|u_{k}(x, t)\right|^{2} d x \\
\leq C \int_{D}|f(x)|^{2} d x+C \int_{0}^{T} \int_{D}\left|V_{k}(x, t)\right|\left|u_{k}(x, t)\right|^{2} d x d t .
\end{aligned}
$$

Taking into account (4.17), we conclude from the previous inequality that

$$
\int_{0}^{T} \int_{D}\left|\nabla u_{k}(x, t)\right|^{2} d x d t \leq C
$$

Here $C$ is independent of $k$.

Now we know that $\left\{u_{k}\right\}$ is a weakly compact sequence in $L^{2}\left(0, T ; W_{0}^{1,2}(D)\right)$. On the other hand we know from (4.6) that $\lim _{k \rightarrow \infty} u_{k}(x, t)=u(x, t)$. Hence it is clear that

$$
u \in L^{2}\left(0, T ; W_{0}^{1,2}(D)\right) .
$$

Next we show that $u \in C^{0}\left([0, T] ; L^{2}(D)\right)$. Since $u_{k}$ is a weak solution of (4.18), we know it is continuous in $D \times(0, T)$. By (4.6), $\lim _{k \rightarrow \infty} u_{k}(x, t)=u(x, t)$ uniformly. Hence $u$ is also continuous in $D \times(0, T)$. Moreover $u$ is bounded because $u_{k}$ is uniformly bounded. Now it is clear that

$$
\lim _{t_{1} \rightarrow t} \int_{D}\left|u\left(x, t_{1}\right)-u(x, t)\right|^{2} d x=0,
$$

which means $u \in C^{0}\left([0, T] ; L^{2}(D)\right)$. This proves (iv) and Theorem A. q.e.d.

Finally we would like to explain why Theorem B is an immediate consequence of Theorem A.

Proof of Theorem B. Following the argument in the proof of Theorem A, we know a weak solution of the initial-boundary value problem is given by

$$
u(x, t)=\int_{D} G(x, t ; y, 0) f(y) d y-\int_{0}^{t} \int_{D} G(x, t ; y, s) g(y, s) d y d s .
$$


Hence we only need to show the uniqueness. More specifically we need to show that the only bounded weak solution to the following problem is zero:

$$
\left\{\begin{array}{l}
\nabla(A \nabla u)-V u-u_{t}=0, \\
u(x, t)=0 \quad \text { when } \quad(x, t) \in \partial D \times[0, T], \\
u(x, 0)=0 .
\end{array}\right.
$$

We can rewrite $(4.21)$ as

$$
\left\{\begin{array}{l}
\nabla(A \nabla u)-u_{t}=V u \\
u(x, t)=0 \quad \text { when } \quad(x, t) \in \partial D \times[0, T], \\
u(x, 0)=0 .
\end{array}\right.
$$

Since $V u \in L^{1}(Q)$, we have that

$$
u(x, t)=-\int_{0}^{t} \int_{D} G_{0}(x, t ; y, s) V(y, s) u(y, s) d y d s .
$$

Here we have used Lemma 6.4 at the end of the paper to justify (4.22). We reassure the reader that the proof of Lemma 6.4 is independent of any arguments that precede it.

Let $M(t)=\sup \{|u(y, s)| \mid(y, s) \in D \times[0, t]\}$. Then (4.22) implies

$$
M(t) \leq C M(t) N_{t}(V) .
$$

Since $V$ is in the parabolic Kato class, $N_{t}(V)<1$ when $t$ is sufficiently small. In this case, (4.23) implies $M(t)=0$. Applying this process repeatedly, we find that $u \equiv 0$. q.e.d.

\section{An upPer AND A LOWER BOUND FOR THE GREen's FUnCtion}

The proof of the Harnack inequality depends heavily on an upper and a lower bound for the Green's function of our equation. These bounds are given in the next few lemmas.

Lemma 5.1. There exist positive constants $C$ and $h_{0}$ such that

$$
|G(x, t ; y, s)| \leq C /(t-s)^{n / 2}, \quad 0<t-s \leq h_{0} .
$$

Proof. We again start with formula (3.1), which is

$$
G(x, t ; y, s)=\sum_{k=0}^{\infty} G_{0} *\left(H G_{0}\right)^{* k}(x, t ; y, s) \equiv \sum_{k=0}^{\infty} J_{k}(x, t ; y, s)
$$

where $H G_{0}(x, t ; y, s) \equiv-V(x, t) G_{0}(x, t ; y, s)$.

We would like to show, for a constant $C_{0}$ and $k=1,2, \ldots$, that

$$
\left|J_{k}(x, t ; y, s)\right| \leq \frac{C_{0}^{k}\left[N_{h}(V)+N_{h}^{*}(V)\right]^{k}}{(t-s)^{n / 2}}, \quad 0<t-s \leq h .
$$

We shall use induction. 
First of all there is a constant $A$ such that

$$
G_{0}(x, t ; y, s) \leq A \frac{1}{(t-s)^{n / 2}} \exp \left(-\alpha \frac{|x-y|^{2}}{t-s}\right) \leq \frac{A}{(t-s)^{n / 2}}
$$

Next

$$
\begin{aligned}
& \left|J_{1}(x, t ; y, s)\right|=\left|\int_{s}^{t} \int_{D} G_{0}(x, t ; z, \tau) V(z, \tau) G_{0}(z, \tau ; y, s) d z d \tau\right| \\
& \leq A^{2} \int_{s}^{t} \int_{D} \frac{1}{(t-\tau)^{n / 2}} \exp \left(-\alpha \frac{|x-z|^{2}}{t-\tau}\right)|V(z, \tau)| \frac{1}{(\tau-s)^{n / 2}} \exp \left(-\alpha \frac{|z-y|^{2}}{\tau-s}\right) d z d \tau \\
& =A^{2} \int_{s}^{\frac{t+s}{2}} \int_{D} \ldots d z d \tau+A^{2} \int_{\frac{t+s}{2}}^{t} \int_{D} \ldots d z d \tau .
\end{aligned}
$$

When $\tau \in\left[s, \frac{t+s}{2}\right], t-\tau \geq(t-s) / 2$; when $\tau \in\left[\frac{t+s}{2}, t\right], \tau-s \geq(t-s) / 2$. Hence

$$
\begin{aligned}
\left|J_{1}(x, t ; y, s)\right| \leq & \frac{A^{2} 2^{n / 2}}{(t-s)^{n / 2}} \int_{s}^{\frac{t+s}{2}} \int_{D}|V(z, \tau)| \frac{1}{(\tau-s)^{n / 2}} \exp \left(-\alpha \frac{|z-y|^{2}}{\tau-s}\right) d z d \tau \\
& +\frac{A^{2} 2^{n / 2}}{(t-s)^{n / 2}} \int_{\frac{t+s}{2}}^{t} \int_{D} \frac{1}{(t-\tau)^{n / 2}} \exp \left(-\alpha \frac{|x-z|^{2}}{t-\tau}\right)|V(z, \tau)| d z d \tau
\end{aligned}
$$

This means

$$
\left|J_{1}(x, t ; y, s)\right| \leq \frac{A^{2} 2^{n / 2}}{(t-s)^{n / 2}}\left[N_{h}(V)+N_{h}^{*}(V)\right]
$$

Now assuming, for a constant $C_{0}$ and when $0<t-s \leq h$,

$$
\left|J_{k}(x, t ; y, s)\right| \leq \frac{C_{0}^{k}\left[N_{h}(V)+N_{h}^{*}(V)\right]^{k}}{(t-s)^{n / 2}},
$$

we get

$$
\begin{aligned}
\left|J_{k+1}(x, t ; y, s)\right|= & \left|\int_{s}^{t} \int_{D} J_{k}(x, t ; z, \tau) V(z, \tau) G_{0}(z, \tau ; y, s) d z d \tau\right| \\
\leq & \int_{s}^{\frac{t+s}{2}} \int_{D}\left|J_{k}(x, t ; z, \tau) V(z, \tau) G_{0}(z, \tau ; y, s)\right| d z d \tau \\
& +\int_{\frac{t+s}{2}}^{t} \int_{D}\left|J_{k}(x, t ; z, \tau) V(z, \tau) G_{0}(z, \tau ; y, s)\right| d z d \tau .
\end{aligned}
$$

When $\tau \in\left[s, \frac{t+s}{2}\right]$, we have, by (5.4),

$$
\left|J_{k}(x, t ; z, \tau)\right| \leq \frac{C_{0}^{k}\left[N_{h}(V)+N_{h}^{*}(V)\right]^{k}}{(t-\tau)^{n / 2}} \leq \frac{2^{n / 2} C_{0}^{k}\left[N_{h}(V)+N_{h}^{*}(V)\right]^{k}}{(t-s)^{n / 2}} ;
$$


when $\tau \in\left[\frac{t+s}{2}, t\right]$ we have $\tau-s \geq(t-s) / 2$, and hence

$$
\left|G_{0}(z, \tau ; y, s)\right| \leq \frac{A}{(\tau-s)^{n / 2}} \leq \frac{2^{n / 2} A}{(t-s)^{n / 2}}
$$

Going back to (5.5) we obtain the inequality

$$
\begin{aligned}
\left|J_{k+1}(x, t ; y, s)\right| & \\
\leq & \frac{2^{n / 2} C_{0}^{k}\left[N_{h}(V)+N_{h}^{*}(V)\right]^{k}}{(t-s)^{n / 2}} \int_{s}^{\frac{t+s}{2}} \int_{D}\left|V(z, \tau) G_{0}(z, \tau ; y, s)\right| d z d \tau \\
& +\frac{2^{n / 2} A}{(t-s)^{n / 2}} \int_{\frac{t+s}{2}}^{t} \int_{D}\left|J_{k}(x, t ; z, \tau) V(z, \tau)\right| d z d \tau \\
\leq & \frac{2^{n / 2} C_{0}^{k}\left[N_{h}(V)+N_{h}^{*}(V)\right]^{k}}{(t-s)^{n / 2}} A N_{h}^{*}(V) \\
& +\frac{2^{n / 2} A}{(t-s)^{n / 2}} \int_{s}^{t} \int_{D}\left|J_{k}(x, t ; z, \tau) V(z, \tau)\right| d z d \tau
\end{aligned}
$$

To estimate the last term of the preceding inequality, let's observe that

$$
\begin{aligned}
& \int_{s}^{t} \int_{D}\left|J_{k}(x, t ; z, \tau) V(z, \tau)\right| d z d \tau \\
& =\int_{s}^{t} \int_{D}\left|\int_{\tau}^{t} \int_{D} J_{k-1}(x, t ; w, l) V(w, l) G_{0}(w, l ; z, \tau) d w d l\right||V(z, \tau)| d z d \tau \\
& \leq \int_{s}^{t} \int_{D} \int_{s}^{l} \int_{D}\left|G_{0}(w, l ; z, \tau) V(z, \tau)\right| d z d \tau\left|J_{k-1}(x, t ; w, l) V(w, l)\right| d w d l \quad \text { (Fubini) } \\
& \leq A N_{h}(V) \int_{s}^{t} \int_{D}\left|J_{k-1}(x, t ; w, l) V(w, l)\right| d w d l .
\end{aligned}
$$

Therefore an easy induction gives us, for $k=1,2, \ldots$,

$$
\int_{s}^{t} \int_{D}\left|J_{k}(x, t ; z, \tau) V(z, \tau)\right| d z d \tau \leq A^{k+1}\left[N_{h}(V)\right]^{k+1} .
$$

Combining (5.6) with (5.7), we deduce that

$$
\begin{aligned}
& \left|J_{k+1}(x, t ; y, s)\right| \\
& \leq \frac{2^{n / 2} C_{0}^{k}\left[N_{h}(V)+N_{h}^{*}(V)\right]^{k}}{(t-s)^{n / 2}} A N_{h}^{*}(V)+\frac{2^{n / 2} A}{(t-s)^{n / 2}} A^{k+1}\left[N_{h}(V)\right]^{k+1} \\
& \leq\left(2^{n / 2} C_{0}^{k} A+2^{n / 2} A^{k+2}\right)\left[N_{h}(V)+N_{h}^{*}(V)\right]^{k+1} \frac{1}{(t-s)^{n / 2}} \\
& \leq C_{0}^{k+1}\left[N_{h}(V)+N_{h}^{*}(V)\right]^{k+1} \frac{1}{(t-s)^{n / 2}} .
\end{aligned}
$$

Here $C_{0}$ is chosen as a number larger than or equal to $2 \max \left\{2^{n / 2} A, 2^{n / 2} A^{2}\right\}$. This proves (5.1). 
The rest of the proof is straightforward. Invoking (3.1) and (5.8), we obtain

$$
|G(x, t ; y, s)| \leq \frac{A}{(t-s)^{n / 2}}+\sum_{k=1}^{\infty} C_{0}^{k}\left[N_{h}(V)+N_{h}^{*}(V)\right]^{k} \frac{1}{(t-s)^{n / 2}} .
$$

Since $V$ belongs to the parabolic Kato class, we can find a positive constant $h_{0}$ such that

$$
C_{0}\left[N_{h}(V)+N_{h}^{*}(V)\right]<1
$$

whenever $0<h \leq h_{0}$. It follows that

$$
|G(x, t ; y, s)| \leq \frac{C}{(t-s)^{n / 2}}, \quad 0<t-s \leq h_{0} .
$$

This proves the lemma. q.e.d.

Based on the method used in Lemma 5.1 we will be able to give a lower bound for the fundamental solution and the Green's function.

Lemma 5.2. Suppose $\Gamma(x, t ; y, s)$ is the fundamental solution of (1.1). Then there is a $C$ such that

$$
\Gamma(x, t ; y, s) \geq \frac{1}{C(t-s)^{n / 2}} \exp \left(-C|x-y|^{2} /(t-s)\right) .
$$

Proof. It is clear that (3.1) and (5.8) remain valid when $G(x, t ; y, s)$ is replaced by $\Gamma(x, t ; y, s)$. Moreover there is a $B>0$ such that

$$
\Gamma_{0}(x, t ; y, s) \geq \frac{B}{(t-s)^{n / 2}}
$$

when $|x-y|^{2} \leq t-s$. This yields, via (3.1) and (5.8),

$$
\Gamma(x, t ; y, s) \geq \frac{B}{(t-s)^{n / 2}}-\sum_{k=1}^{\infty} C_{0}^{k}\left[N_{h}(V)+N_{h}^{*}(V)\right]^{k} \frac{1}{(t-s)^{n / 2}} .
$$

We can therefore take a constant $h$ so that

$$
\Gamma(x, t ; y, s) \geq \frac{B}{2(t-s)^{n / 2}}
$$

whenever $|x-y|^{2} \leq t-s$ and $0<t-s \leq h$.

As indicated in [FS2] (cf. Theorem 2.7), an estimate such as (5.12) implies the desired lower bound. We refer the reader to that source for details. q.e.d.

In what follows, $G^{(\xi, R)}(x, t ; y, s)$ denotes the Green's function of (1.1) with zero boundary data on $\partial B(\xi, R) \times(0, \infty)$. 
Lemma 5.3. For each $\delta, \gamma \in(0,1)$ there is an $\epsilon>0$ and $R_{0}>0$ such that

$$
G^{(\xi, R)}(x, t ; y, s) \geq \frac{\epsilon}{|B(\xi, \delta R)|}
$$

for all, $x, y \in B(\xi, \delta R)$ and $s<t$ satisfying $\gamma R^{2} \leq t-s \leq R^{2} \leq R_{0}^{2}$.

Proof. The proof of the lemma is a copy of that of Lemma 5.2.

By Lemma 5.1 in [FS2],

$$
G_{0}^{(\xi, R)}(x, t ; y, s) \geq \frac{\epsilon}{|B(\xi, \delta R)|} \geq \frac{C}{(t-s)^{n / 2}} .
$$

Using (3.1) and (5.8) we obtain

$$
G^{(\xi, R)}(x, t ; y, s) \geq \frac{C}{(t-s)^{n / 2}}-\sum_{k=1}^{\infty} C_{0}^{k}\left[N_{R^{2}}(V)+N_{R^{2}}^{*}(V)\right]^{k} \frac{1}{(t-s)^{n / 2}} .
$$

When $R$ is sufficiently small we know that

$$
\sum_{k=1}^{\infty} C_{0}^{k}\left[N_{R^{2}}(V)+N_{R^{2}}^{*}(V)\right]^{k}<C / 2
$$

for all $t, s$ such that $0<t-s \leq R^{2}$. Hence

$$
G^{(\xi, R)}(x, t ; y, s) \geq \frac{C}{2(t-s)^{n / 2}} .
$$

q.e.d.

\section{Proof of Theorem C: The Harnack Inequality}

In the following we use the notation $m(R)$ and $M(R)$ to denote, respectively, the minimum and maximum values of $u=u(x, t)$ on $B(\xi, R) \times\left[s-R^{2}, s\right]$. In the next lemma we will make the additional assumption that $V \in L^{\infty}$. However as demonstrated in the proof, all the constants involved are independent of the $L^{\infty}$ norm of $V$. This independence is crucial in the proof of Theorem $\mathrm{C}$ later on, when we will remove the extra assumption on $V$ via a limiting argument.

Lemma 6.1. Suppose $u$ is a non-negative weak solution of (1.1). For each $\delta \in$ $(0,1)$ there is a $\rho \in(0,1)$ such that for all $(\xi, s) \in \mathbf{R}^{n} \times \mathbf{R}$ :

$$
O s c(u ; \xi, s, \delta R) \equiv M(\delta R)-m(\delta R) \leq \rho M(R)
$$

whenever $R$ is sufficiently small.

Proof. Set $S=\left\{x \in B(\xi, \delta R) \mid u\left(x, s-R^{2}\right) \geq(M(R)+m(R)) / 2\right\}$.

Case 1. Assume that $|S| /|B(\xi, \delta R)| \geq 1 / 2$.

By Lemma 5.3, when $(x, t) \in B(\xi, \delta R) \times\left[s-\delta^{2} R^{2}, s\right]$,

$$
\int_{B(\xi, R)} G^{(\xi, R)}\left(x, t ; y, s-R^{2}\right) d y \geq \frac{\epsilon|B(\xi, \delta R)|}{|B(\xi, \delta R)|} \geq \epsilon .
$$


Hence for the same $(x, t)$,

$$
\begin{aligned}
u(x, t)-\epsilon m(R) & \geq \int_{B(\xi, R)}\left(u\left(y, s-R^{2}\right)-m(R)\right) G^{(\xi, R)}\left(x, t ; y, s-R^{2}\right) d y \\
& \geq \int_{S}\left(u\left(y, s-R^{2}\right)-m(R)\right) G^{(\xi, R)}\left(x, t ; y, s-R^{2}\right) d y \\
& \geq \frac{M(R)-m(R)}{2} \int_{S} G^{(\xi, R)}\left(x, t ; y, s-R^{2}\right) d y \\
& \geq \frac{M(R)-m(R)}{2} \frac{\epsilon|S|}{|B(\xi, \delta R)|} \\
& \geq \frac{\epsilon}{4}(M(R)-m(R))
\end{aligned}
$$

and so

$$
m(\delta R) \geq \frac{\epsilon}{4} M(R)+\frac{3 \epsilon}{4} m(R) .
$$

It follows that

$$
M(\delta R)-m(\delta R) \leq M(R)-m(\delta R) \leq\left(1-\frac{\epsilon}{4}\right) M(R)-\frac{3 \epsilon}{4} m(R),
$$

which implies

$$
M(\delta R)-m(\delta R) \leq\left(1-\frac{\epsilon}{4}\right) M(R) .
$$

In other words, we can take $\rho=1-\frac{\epsilon}{4}$.

Case 2. Assume that $|S| /|B(\xi, \delta R)| \leq 1 / 2$.

First we observe that $M(R)-u$ is a non-negative weak solution of the equation

$$
H(M(R)-u)=-V M(R) .
$$

Hence for $(x, t) \in B(\xi, \delta R) \times\left[s-\delta^{2} R^{2}, s\right]$,

$$
\begin{aligned}
M(R)-u(x, t) \geq & \int_{B(\xi, R)}\left(M(R)-u\left(y, s-R^{2}\right)\right) G^{(\xi, R)}\left(x, t ; y, s-R^{2}\right) d y \\
& \quad+\int_{s-R^{2}}^{t} \int_{B(\xi, R)} V(z, \tau) M(R) G^{(\xi, R)}(x, t ; z, \tau) d z d \tau .
\end{aligned}
$$

We need to control the second term of the right-hand side of (6.3). According to (3.1) and (5.7), we can find a positive constant $b$ such that

$$
\begin{aligned}
\mid \int_{s-R^{2}}^{t} \int_{B(\xi, R)} & V(z, \tau) G^{(\xi, R)}(x, t ; z, \tau) d z d \tau \mid \\
& \leq \int_{t-2 R^{2}}^{t} \int_{B(\xi, R)}\left|V(z, \tau) G^{(\xi, R)}(x, t ; z, \tau)\right| d z d \tau \\
& \leq \sum_{k=0}^{\infty} \int_{t-2 R^{2}}^{t} \int_{B(\xi, R)}\left|J_{k}(x, t ; z, \tau) V(z, \tau)\right| d z d \tau \\
& \leq \sum_{k=0}^{\infty} A^{k+1}\left[N_{2 R^{2}}(V)\right]^{k+1} \leq b
\end{aligned}
$$


when $R$ is sufficiently small. Moreover we can choose $R$ so small that

$$
0<b<\epsilon / 8
$$

Hence (6.3) yields

$$
M(R)-u(x, t) \geq \int_{B(\xi, R)}\left(M(R)-u\left(y, s-R^{2}\right)\right) G^{(\xi, R)}\left(x, t ; y, s-R^{2}\right) d y-b M(R),
$$

which shows that

$$
\begin{aligned}
(1+b) M(R) & -u(x, t) \geq \int_{B(\xi, R)}\left(M(R)-u\left(y, s-R^{2}\right)\right) G^{(\xi, R)}\left(x, t ; y, s-R^{2}\right) d y \\
& \geq \int_{B(\xi, \delta R)-S}\left(M(R)-u\left(y, s-R^{2}\right)\right) G^{(\xi, R)}\left(x, t ; y, s-R^{2}\right) d y \\
& \geq \frac{M(R)-m(R)}{2} \int_{B(\xi, \delta R)-S} G^{(\xi, R)}\left(x, t ; y, s-R^{2}\right) d y \\
& \geq \frac{M(R)-m(R)}{2} \frac{\epsilon|B(\xi, \delta R)-S|}{|B(\xi, \delta R)|} \\
& \geq \frac{\epsilon}{4}(M(R)-m(R))
\end{aligned}
$$

and so

$$
-M(\delta R) \geq\left(\frac{\epsilon}{4}-1-b\right) M(R)-\frac{\epsilon}{4} m(R)
$$

and

$$
M(\delta R) \leq\left(1+b-\frac{\epsilon}{4}\right) M(R)+\frac{\epsilon}{4} m(R)
$$

By (6.4), we have

$$
M(\delta R) \leq\left(1-\frac{\epsilon}{8}\right) M(R)+\frac{\epsilon}{4} m(R) .
$$

Therefore

$$
\begin{aligned}
M(\delta R)-m(\delta R) & \leq M(\delta R)-m(R) \\
& \leq\left(1-\frac{\epsilon}{8}\right) M(R)-\left(1-\frac{\epsilon}{4}\right) m(R) \\
& \leq\left(1-\frac{\epsilon}{8}\right) M(R) .
\end{aligned}
$$

Taking $\rho=1-\frac{\epsilon}{8}$, we complete the proof. q.e.d.

We are now ready to prove a special case of the Harnack inequality. Except for some modifications the proof is the same as that of Theorem 5.4 in [FS2], but for completeness we reproduce it in the sequel.

Theorem 6.2 (Harnack inequality, a special case). In addition to the hypothesis of Theorem $C$ we assume that $V \in L^{\infty}(Q)$. Let $0<\alpha<\beta<1$ and $\delta \in(0,1)$ be given. Then there exist $M>0$ and $R_{0}>0$ such that for all $(x, s) \in \mathbf{R}^{n} \times \mathbf{R}$, all positive $R<R_{0}$ and all non-negative $u \in C^{\infty}\left(B(x, R) \times\left[s-R^{2}, s\right]\right)$ satisfying $H u=0$, one has

$$
u(y, t) \leq M u(x, s)
$$


for all $(y, t) \in B(x, \delta R) \times\left[s-\beta R^{2}, s-\alpha R^{2}\right]$. Here all the constants depend on the rate of convergence to zero of $N_{h}(V)+N_{h}^{*}(V)$ when $h$ approaches zero, but they do not depend on the $L^{\infty}$ norm of $V$.

Proof. By translation and rescaling we may and will assume that $(x, s)=(0,0)$ and $R=1$. Also, we assume that $u(0,0)=1$.

From Lemma 5.3 we know that there is an $\epsilon>0$ such that for all $r \in[-1, \alpha]$ and $\lambda>0$ :

$$
\begin{aligned}
1 & =u(0,0) \geq \int G^{(0,1)}(0,0 ; \eta, r) u(\eta, r) d \eta \\
& \geq \epsilon \lambda|S(r, \lambda)|
\end{aligned}
$$

where $S(r, \lambda) \equiv\left\{\eta \in B\left(0, \frac{1}{2}(1+\delta)\right) \mid u(\eta, r) \geq \lambda\right\}$.

Next let $\rho$ be the constant in Lemma 6.1 and set $\sigma=\frac{1}{2}(1-\rho)$ and $K=\frac{1}{2}(1+1 / \rho)$. Also define $r(\lambda)=\left(2 / \Omega_{n} \epsilon \sigma \lambda\right)^{1 / n}$ for $\lambda>0$, where $\Omega_{n}=|B(0,1)|$. Now suppose that $(y, t) \in B\left(0, \frac{1}{2}(1+\delta)\right) \times(-1, \alpha)$ and $\lambda>0$ are such that $u(y, t) \geq \lambda$ and $B(y, 2 r(\lambda)) \times\left[t-4 r(\lambda)^{2}, t\right] \subset B\left(0, \frac{1}{2}(1+\delta)\right) \times[-1, \alpha]$.

Since, for $r \in[-1, \alpha],|S(r, \lambda \sigma)| \leq 1 / \epsilon \sigma \lambda$ and $|B(y, r(\lambda))|=2 / \epsilon \sigma \lambda$, there is an $\eta \in B(y, r(\lambda))$ such that $u(\eta, r)<\sigma \lambda$. Hence $O s c(u ; y, t, r(\lambda)) \geq u(y, t)-u(\eta, r)>$ $(1-\sigma) \lambda$; and so, by Lemma 6.1 , there is a $\left(y^{\prime}, t^{\prime}\right) \in B(y, 2 r(\lambda)) \times\left[t-4 r(\lambda)^{2}, t\right]$ such that $u\left(y^{\prime}, t^{\prime}\right) \geq \frac{1}{\rho} O s c(u ; y, t, r(\lambda))>\frac{1}{\rho}(1-\sigma) \lambda \equiv K \lambda$.

Finally, define $M$ by the relation

$$
r(M)=\frac{1}{2}(1-\beta)(1-\delta)\left(1-1 / K^{1 / n}\right) ;
$$

and suppose that there were a $(y, t) \in B(0, \delta) \times[-\beta,-\alpha]$ such that $u(y, t) \geq M$. Then by the preceding paragraph, we could inductively find $\left(y_{m}, t_{m}\right), m \geq 0$, such that $\left(y_{0}, t_{0}\right)=(y, t)$, and $\left(y_{m}, t_{m}\right) \in B\left(y, 2 r\left(K^{m} M\right)\right) \times\left[t_{m}-4 r\left(K^{m} M\right)^{2}, t_{m}\right] \subset$ $B\left(0, \frac{1}{2}(1+\delta)\right) \times[-1, \alpha]$, and $u\left(y_{m}, t_{m}\right) \geq K^{m} M$. But this would mean that $u$ is unbounded in $B\left(0, \frac{1}{2}(1+\delta)\right) \times[-1, \alpha]$, and so no such $(y, t)$ exists. q.e.d.

Now we are ready to give the

Proof of Theorem C (Harnack inequality). Note that Theorem 6.2 is not in its most general form, since $V$ needs to be an $L^{\infty}$ function. However, to remove this extra assumption and to obtain the Harnack inequality in its entirety (Theorem C) we only need the following lemma about approximation. If we assume for the moment that the lemma is true, we have thus completed the proof Theorem C. q.e.d.

Lemma 6.3. Suppose $u$ is a weak solutoin of equation (1.1) in $D \times[0, T]$. Then $u$ is the $L^{1}$-local limit of $\left\{u_{m}\right\}$, where $u_{m}$ is a weak solution of (1.1) in which $V$ is replaced by $V_{m}$. Here $V_{m} \in L^{\infty}(D \times[0, T])$ and $V_{m} \rightarrow V$ a.e.

Proof. According to the definition of weak solutions,

$$
u \in C^{0}\left([0, T] ; L^{2}(D)\right) \cap L^{2}\left(0, T ; W^{1,2}(D)\right) .
$$

Choose a smooth subdomain $D_{1}$ and $a, b$ such that $\bar{D}_{1} \times[a, b] \subset D \times(0, T)$. Let $\eta \in C_{0}^{\infty}(D \times[0, T])$ be such that $\eta(x, t)=1$ for $(x, t) \in D_{1} \times[a, b]$. Then $\eta u \in L^{2}\left(0, T ; W_{0}^{1,2}(D)\right)$ satisfies

$$
\left\{\begin{array}{l}
H(\eta u) \equiv \nabla(A \nabla(\eta u))-V \eta u-(\eta u)_{t}=f, \\
\eta u(x, t)=0 \quad \text { when } \quad(x, t) \in \partial D \times[0, T], \\
\eta u(x, 0)=0,
\end{array}\right.
$$


where $f \equiv \nabla(A(\nabla \eta) u)+(\nabla \eta) A \nabla u-u \eta_{t}$. Evidently $f \in L^{2}\left(0, T ; W^{-1,2}(D)\right)$.

Now let us consider the following problem:

$$
\left\{\begin{array}{l}
H_{m}\left(u_{m}\right) \equiv \nabla\left(A \nabla u_{m}\right)-V_{m} u_{m}-\left(u_{m}\right)_{t}=f \\
u_{m}(x, t)=0 \quad \text { when } \quad(x, t) \in \partial D \times[0, T] \\
u_{m}(x, 0)=0
\end{array}\right.
$$

According to Theorem 40.1 in $[\mathrm{T}]$ and since $V_{m} \in L^{\infty}(D \times[0, T])$, problem (6.6) is well-posed in $C^{0}\left[(0, T) ; L^{2}(D)\right] \cap L^{2}\left(0, T ; W_{0}^{1,2}(D)\right)$. Comparing (6.5) with (6.6), we know that $u_{m}-\eta u$ satisfies

$$
\left\{\begin{array}{l}
\nabla\left(A \nabla\left(u_{m}-\eta u\right)\right)-\left(u_{m}-\eta u\right)_{t}-V_{m}\left(u_{m}-\eta u\right)=\left(V_{m}-V\right) \eta u \\
\left(u_{m}-\eta u\right)(x, t)=0, \quad \text { when } \quad(x, t) \in \partial D \times[0, T] \\
\left(u_{m}-\eta u(x, 0)\right)=0,
\end{array}\right.
$$

in the weak sense. By the next lemma, which will be stated and proved subsequently,

$$
\left(u_{m}-\eta u\right)(x, t)=-\int_{0}^{t} \int_{D} G_{m}(x, t ; y, s)\left(V_{m}(y, s)-V(y, s)\right) \eta u(y, s) d y d s .
$$

By Proposition 3.3, there is a constant $C$ independent of $m$ or $(y, s)$ such that

$$
\int_{s}^{T} \int_{D}\left|G_{m}(x, t ; y, s)\right| d x d t \leq C .
$$

Hence (6.8) implies

$$
\int_{0}^{T} \int_{D}\left|u_{m}(x, t)-\eta u(x, t)\right| d x d t \leq C \int_{0}^{T} \int_{D}\left|\left(V_{m}(y, s)-V(y, s)\right) u(y, s)\right| d y d s .
$$

The right-hand side of the preceding inequality converges to zero by the dominated convergence theorem because $V u \in L^{1}(D \times[0, T])$ and $\left|V_{m}\right| \leq|V|$.

Note that $\left.f\right|_{D_{1} \times[a, b]}=0$. Hence $u_{m}$ is a weak solution of $H_{m}\left(u_{m}\right)=0$ in $D_{1} \times[a, b]$. Moreover $u_{m} \rightarrow u$ a.e. in $D_{1} \times[a, b]$. So except for verifying (6.8), we have thus proved Lemma 6.3. q.e.d.

Equation (6.8) is a direct consequence of the following Lemma 6.4, whose proof is independent of any arguments we have made so far.

Lemma 6.4. Assume that $V \in L^{\infty}(Q)$ and $f \in L^{1}(Q)$, and suppose

$$
u \in C^{0}\left([0, T] ; L^{2}(D)\right) \cap L^{2}\left(0, T ; W_{0}^{1,2}(D)\right)
$$

is a weak solution of

$$
\left\{\begin{array}{l}
L u \equiv \nabla(A \nabla u)-V u-u_{t}=f \\
u(x, t)=0, \quad \text { when } \quad(x, t) \in \partial D \times[0, T] \\
u(x, 0)=0 .
\end{array}\right.
$$


Then

$$
u(x, t)=-\int_{0}^{t} \int_{D} G(x, t ; y, s) f(y, s) d y d s,
$$

where $G$ is the Green's function of $L$ in $Q$.

Proof. The result in this lemma may be known. But since we are unable to find an exact source, we give a proof for the sake of completeness.

Using the notation $w(x, t)=-\int_{0}^{t} \int_{D} G(x, t ; y, s) f(y, s) d y d s$, we need to show that $u(x, t)=w(x, t)$.

Given $\psi \in C^{\infty}(\bar{Q})$, we first consider the following initial-boundary value problem for the conjugate operator of $L$ :

$$
\left\{\begin{array}{l}
\nabla(A \nabla \phi)-V \phi+\phi_{t}=\psi, \\
\phi(x, t)=0, \quad \text { when } \quad(x, t) \in \partial D \times[0, T], \\
\phi(x, T)=0 .
\end{array}\right.
$$

By the standard theory in $[\mathrm{T}]$, this problem has a unique solution $\phi$ satisfying

$$
\phi \in L^{2}\left(0, T ; W_{0}^{1,2}(D)\right), \quad \phi_{t} \in L^{2}\left(0, T ; W^{-1,2}(D)\right) .
$$

This implies that $\phi \in C^{0}\left([0, T] ; L^{2}(D)\right), \phi \in L^{\infty}(Q)$ and

$$
\phi(y, s)=-\int_{s}^{T} \int_{D} G(x, t ; y, s) \psi(x, t) d x d t .
$$

For simplicity we write $\phi=M^{*}(\psi)$.

Now using the definition of $w$ and Fubini's theorem, we have

$$
\begin{aligned}
& \int_{0}^{T} \int_{D} w(x, t) \psi(x, t) d x d t \\
& =-\int_{0}^{T} \int_{D} \int_{0}^{t} \int_{D} G(x, t ; y, s) f(y, s) d y d s \psi(x, t) d x d t \\
& =-\int_{0}^{T} \int_{D} \int_{s}^{T} \int_{D} G(x, t ; y, s) \psi(x, t) d x d t f(y, s) d y d s \\
& =\int_{0}^{T} \int_{D} M^{*}(\psi)(y, s) f(y, s) d y d s .
\end{aligned}
$$

In short,

$$
\int_{0}^{T} \int_{D} w \psi d x d t=\int_{0}^{T} \int_{D} M^{*}(\psi) f d x d t
$$

for all $\psi \in C^{\infty}(\bar{Q})$.

Next we turn our attention to $u$. We wish to show that

$$
\int_{0}^{T} \int_{D} u \psi d x d t=\int_{0}^{T} \int_{D} M^{*}(\psi) f d x d t
$$


for all $\psi \in C^{\infty}(\bar{Q})$. Once (6.13) is established, from it and (6.12) we conclude that $u \equiv w$ a.e.

The proof of (6.13) starts with the following observation. From (6.9), by the definition of weak solutions, for all $\eta \in C^{\infty}(\bar{Q})$ such that $\eta=0$ on $(\partial D \times[0, T]) \cup$ $(D \times\{T\})$,

$$
\int_{0}^{T} \int_{D}\left[-A \nabla u \nabla \eta-V u \eta+u \eta_{t}\right] d x d t=\int_{0}^{T} \int_{D} f \eta d x d t
$$

Choose a sequence of positive definite matrices $\left\{A_{m}\right\}$ of $C^{\infty}$ functions and a sequence $\left\{V_{m}\right\}$ of $C^{\infty}$ functions such that $A_{m} \rightarrow A$ a.e. and $V_{m} \rightarrow V$ a.e. respectively. Let $\eta_{m}$ be the unique $C^{\infty}(\bar{Q})$ solution of the following problem:

$$
\left\{\begin{array}{l}
\nabla\left(A_{m} \nabla \eta_{m}\right)-V_{m} \eta_{m}+\left(\eta_{m}\right)_{t}=\psi, \\
\eta_{m}(x, t)=0, \quad \text { when } \quad(x, t) \in \partial D \times[0, T], \\
\eta_{m}(x, T)=0 .
\end{array}\right.
$$

Obviously (6.14) still holds when $\eta$ is replaced by $\eta_{m}$, i.e.

$$
\int_{0}^{T} \int_{D}\left[-A \nabla u \nabla \eta_{m}-V u \eta_{m}+u\left(\eta_{m}\right)_{t}\right] d x d t=\int_{0}^{T} \int_{D} f \eta_{m} d x d t
$$

or equivalently

$$
\left\langle u, \nabla\left(A \nabla \eta_{m}\right)-V \eta_{m}+\left(\eta_{m}\right)_{t}\right\rangle=\int_{0}^{T} \int_{D} f \eta_{m} d x d t .
$$

Here $\langle.,$.$\rangle means the pairing of an element in L^{2}\left(0, T ; W_{0}^{1,2}(D)\right)$ with an element in the dual space $L^{2}\left(0, T ; W^{-1,2}(D)\right)$. We remark that $\left\|\eta_{m}\right\|_{L^{\infty}(Q)}$ is bounded independently of $m$.

Now suppose that $\phi$ is the function given by (6.10), then

$$
\left\{\begin{array}{l}
\nabla\left(A_{m} \nabla\left(\eta_{m}-\phi\right)\right)-V_{m}\left(\eta_{m}-\phi\right)+\left(\eta_{m}-\phi\right)_{t} \\
\quad=\nabla\left(\left(A-A_{m}\right) \nabla \phi\right)+\left(V_{m}-V\right) \phi, \\
\left(\eta_{m}-\phi\right)(x, t)=0, \quad \text { when } \quad(x, t) \in \partial D \times[0, T], \\
\left(\eta_{m}-\phi\right)(x, T)=0 .
\end{array}\right.
$$

According to the theory in $[\mathrm{T}]$, there is a constant $C$ independent of $m$ such that

$$
\begin{aligned}
\| \eta_{m}-\phi & \left\|_{L^{2}\left(0, T ; W^{1,2}(D)\right)}+\right\|\left(\eta_{m}-\phi\right)_{t} \|_{L^{2}\left(0, T ; W^{-1,2}(D)\right)} \\
& \leq C\left\|\nabla\left(\left(A-A_{m}\right) \nabla \phi\right)+\left(V_{m}-V\right) \phi\right\|_{L^{2}\left(0, T ; W^{-1,2}(D)\right)} \\
& \leq C\left\|\left(A-A_{m}\right) \nabla \phi\right\|_{L^{2}(Q)}+C\left\|\left(V_{m}-V\right) \phi\right\|_{L^{2}(Q)} \rightarrow 0
\end{aligned}
$$

Combining (6.16) with (6.18), we obtain

$$
\left\langle u, \nabla(A \nabla \phi)-V \phi+\phi_{t}\right\rangle=\int_{0}^{T} \int_{D} f \phi d x d t .
$$


Finally, from (6.10),

$$
\int_{0}^{T} \int_{D} u \psi d x d t=\left\langle u, \nabla(A \nabla \phi)-V \phi+\phi_{t}\right\rangle=\int_{0}^{T} \int_{D} f \phi d x d t d x d t .
$$

This proves (6.13) and the lemma. q.e.d.

Remark. It is a pleasure to thank the referee for observing that condition $\mathrm{K}$ can be relaxed to

$$
\lim _{h \rightarrow 0} \sup \left[N_{h}(V)+N_{h}^{*}(V)\right] \leq \epsilon
$$

for some sufficiently small constant $\epsilon$.

We conclude the paper by posing an open question.

Question: Does condition $\mathrm{K}$ on $V$ imply a Gaussian upper bound for the fundamental solution of (1.1)? In other words, are there constants $C_{1}, C_{2}$ depending on $T$ such that

$$
\Gamma(x, t ; y, s) \leq \frac{C_{1}}{(t-s)^{n / 2}} \exp \left(-C_{2} \frac{|x-y|^{n}}{t-s}\right) ?
$$

Here $x, y \in \mathbf{R}^{n}$ and $0<t-s \leq T$.

\section{REFERENCES}

[A] D.G. Aronson, Non-negative solutions of linear parabolic equations, Annali della Scuola Norm. Sup. Pisa XXII (1968), 607-694. MR 55:8553

[AS] M. Aizenman and B. Simon, Brownian motion and Harnack's inequality for Schrödinger operators, Comm. Pure Appl. Math 35 (1982), 209-271. MR 84a:35062

[CFG] F. Chiarenza, E. Fabes and N. Garofalo, Harnack's inequality for Schrödinger operators and the continuity of solutions, Proc. Amer. Math. Soc. 307 (1986), 415-425. MR 88a:35037

[CZ] M. Cranston and Z. Zhao, Conditional transformation of drift formula and potential theory for $\frac{1}{2} \Delta+b(.) \nabla$., Comm. Math. Phys. 112 (1987), 613-625. MR 89g:35030

[CFZ] M.Cranston, E.Fabes, Z.Zhao, Conditional gauge and potential theory for the Schrödinger operator, Trans. Amer. Math. Soc. 307 (1988), 171-194. MR 90a:60135

[FS1] E.B. Fabes and D.W. Stroock, The $L^{p}$-integrability of Green's functions and fundamental solutions for elliptic and parabolic equations, Duke Math. J. 51 (1984), 997-1016. MR 86g:35057

[FS2] E.B. Fabes and D.W. Stroock, A new proof of Moser's parabolic Harnack inequality using the old idea of Nash, Arch. Rational Mech. Anal. 96 (1986), 327-338. MR 88b:35037

[GW] M. Gruter and K.O. Widman, The Green function for uniformly elliptic equations, Manu. Math. 37 (1982), 303-342. MR 83h:35033

[S] K. Sturm, Harnack's inequality for parabolic operators with singular low order terms, Math. Z. 216 (1994), 593-612. MR 95g:35027

[T] F. Treves, Basic Linear Partial Differential Equations, Academic Press, 1975. MR56:6063

Department of Mathematics, Purdue University, West Lafayette, Indiana 47907

E-mail address: zhangq@math.purdue.edu 